\title{
Electron beam-based sources of ultrashort x-ray pulses
}

\author{
Alexander Zholents \\ Advanced Photon Source, Argonne National Laboratory \\ Argonne, IL 60439
}

(September 7, 2010)

The submitted manuscript has been created by UChicago Argonne, LLC, Operator of
Argonne National Laboratory (“Argonne”). Argonne, a U.S. Department of Energy
Office of Science laboratory, is operated under Contract No. DE-AC02-06CH11357.
The U.S. Government retains for itself, and others acting on its behalf, a paid-up
nonexclusive, irrevocable worldwide license in said article to reproduce, prepare
derivative works, distribute copies to the public, and perform publicly and display
publicly, by or on behalf of the Government.

To be published in Reviews of Accelerator Science and Technology 


\title{
Electron beam-based sources of ultrashort x-ray pulses*
}

\author{
Alexander Zholents \\ Argonne National Laboratory, Advanced Photon Source, \\ 9700 South Cass Ave., Argonne, IL 60439
}

\begin{abstract}
A review of various methods for generation of ultrashort x-ray pulses using relativistic electron beam from conventional accelerators is presented. Both spontaneous and coherent emission of electrons is considered.
\end{abstract}

\section{Introduction}

The importance of the time-resolved studies of matter at picosecond (ps), femtosecond (fs), and atttosecond (as) time scales using $\mathrm{x}$-rays has been widely recognized including by award of a Nobel Prize in 1999 [Zewa]. Extensive reviews of scientific drivers can be found in [BES1, BES2, BES3, Lawr, Whit]. Several laser-based techniques have been used to generate ultrashort x-ray pulses including laser-driven plasmas [Murn, Alte, Risc, Rose, Zamp], high-order harmonic generation [Schn, Rund, Wang, Arpi], and laser-driven anode sources [Ande]. In addition, ultrafast streak-camera detectors have been applied at synchrotron sources to achieve temporal resolution on the picosecond time scale [Wulf, Lind1].

In this paper, we focus on a different group of techniques that are based on the use of the relativistic electron beam produced in conventional accelerators. In the first part

\footnotetext{
* Work supported by the U. S. Department of Energy, Office of Science, Office of Basic Energy Sciences, under Contract No. DE-AC02-06CH11357.
} 
we review several techniques that utilize spontaneous emission of electrons and show how solitary sub-ps x-ray pulses can be obtained at existing storage ring based synchrotron light sources and linacs. In the second part we consider coherent emission of electrons in the free-electron lasers (FELs) and review several techniques for a generation of solitary sub-fs x-ray pulses. Remarkably, the x-ray pulses that can be obtained with the FELs are not only significantly shorter than the ones considered in Part 1, but also carry more photons per pulse by many orders of magnitude.

\section{Part 1: Spontaneous emission}

\section{Generation of Ultrashort X-ray Pulses from an Electron Storage Ring}

\subsection{Preamble}

Modern synchrotron light sources based on electron storage rings operate with electron bunches whose rms bunch length in the zero-current approximation is defined by the total gap voltage $V$ of the radio frequency (rf) accelerating cavities, harmonic number $h$ of the rf field, electron bunch energy spread $\sigma_{E}$, and momentum compaction factor $\alpha_{c}$ :

$$
\sigma_{z, 0}=\frac{\sigma_{E}}{E} c T_{r} \sqrt{\frac{\alpha_{c} E_{b}}{2 \pi h V \cos \phi_{s}}} .
$$

Here $E_{b}$ is the electron beam energy, $T_{r}$ is the revolution time and $\phi_{s}$ is the synchronous phase of the rf field, $c$ is the speed of light, and $e$ is the electron charge. Typically $\sigma_{z, 0} / \mathrm{c}$ is of the order of a few tens of ps. However, as the electron beam current increases to a few mA per bunch, the bunch length also increases due to impact of the self-induced fields [Pell, Bane] and microwave instability [Gao, Chao]. Therefore, most of the light sources operate with electron bunches whose length is greater than $\sigma_{z, 0}$. Several 
approaches to shorten the electron bunch have been tried, and one that takes advantage of a small (close to a zero) $\alpha_{c}$ had been found to be the most successful [Feik1, Feik2]. However, the synchrotron tune

$$
v_{s}=\frac{1}{2 \pi} \sqrt{\frac{\alpha_{c} e \dot{V}}{E_{b}}}
$$

also decreases with $\alpha_{c}$, and less frequent change of particle positions inside the electron bunch leaves more time for instabilities to build up. As a result, short bunches of the order of 1 ps can only be obtained along with a dramatic reduction of the electron bunch current [Feik1, Limb]. This seems to be acceptable for generation of coherent synchrotron radiation (CSR) in the THz part of the radiation spectrum [Wüst] but not for spontaneous emission of photons in the $\mathrm{x}$-ray part of the spectrum. Also, the lattice of $\mathrm{x}$ ray sources is always optimized to yield the smallest electron beam emittance, but low $\alpha_{c}$ storage rings need a negative dispersion function in a large number of bending magnets, and this is incompatible with a lowest-emittance lattice.

Up to this point we presumed that the x-ray pulse should have the same lengths as the electron bunch. However, one can obtain a much shorter x-ray pulse if one can select the radiation emitted by electrons from a short section of the electron bunch and separate it from the radiation of all other electrons. One way to achieve this is to use ultrafast streak camera detectors [Wulf, Lind1]. Another way is to force an ultrashort slice of the electron bunch to emit photons in a different direction than other electrons. Two variants of the latter approach will be discussed next. 


\subsection{Laser Energy Modulation of Electron Bunches}

The "slicing” technique proposed in [Zhol1] uses a femtosecond optical pulse to generate sub-ps $\mathrm{x}$-rays pulses. Figure 1 shows a schematic of this technique. A femtosecond optical pulse of moderate energy $(\sim 1 \mathrm{~mJ})$ modulates the energy of an ultrashort slice of a stored electron bunch as they co-propagate through a wiggler (Fig. 1a). The energy-modulated electrons within this slice are spatially separated from the main bunch in a dispersive section of the storage ring (Fig. 1b) and can then be used to generate femtosecond x-rays (Fig. 1c) at a bend-magnet (or insertion-device) beamline. Note that energy modulation of an ultrashort slice will leave behind a hole or dark pulse in the main electron bunch (see Fig.1c). This will be manifested in the generated x-rays and, in principle, can be used for time-resolved spectroscopy in the same manner as a bright pulse. The original electron bunch is recovered due to synchrotron radiation damping, leaving no impact from energy modulation on the electron beam lifetime.

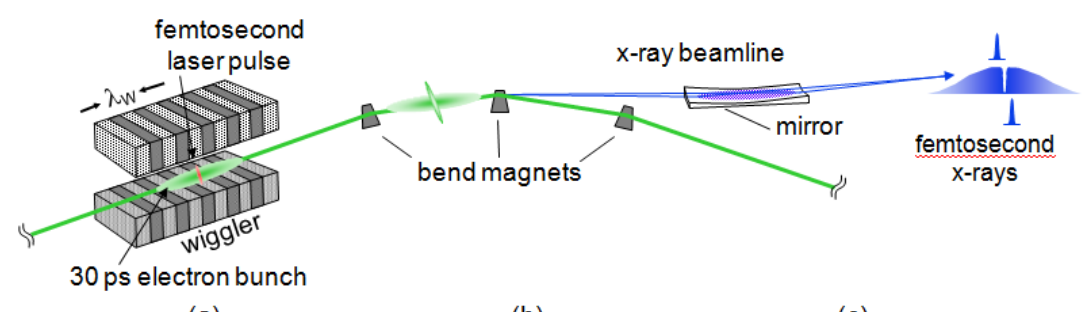

(a)

(b)

(c)

Figure 1. Schematic of the laser slicing method for generating sub-ps synchrotron pulses.

Effective energy modulation of the electrons is accomplished using the high peak electric field $\left(\sim 10^{9} \mathrm{~V} / \mathrm{m}\right)$ of a femtosecond laser pulse. Electrons that co-propagate with the optical pulse through a wiggler are accelerated or decelerated depending on the optical phase $\phi$, as seen by each electron at the entrance of the wiggler. The energy 
exchange between the optical field and the electron is at maximum when the central wavelength of the spontaneous emission from an electron passing through the wiggler, given by

$$
\lambda_{S}=\frac{\lambda_{W}}{2 \gamma^{2}}\left(1+K^{2} / 2^{2}+(\gamma \theta)^{2}\right),
$$

satisfies the resonance condition $\lambda_{S}=\lambda_{L}$, where $\lambda_{L}$ is the laser wavelength, $\lambda_{W}$ is the wiggler period, $\gamma$ is the Lorentz factor, $\theta$ is the angle of observation relative to the beam axis, and the deflection parameter $K=e B_{0} \lambda_{W} / 2 \pi m c$ is the normalized vector potential of the wiggler magnetic field $B_{0}$. We note that the maximum spectral intensity of the electron wiggler emission integrated over the solid angle is red shifted relative to a zero angle emission. Therefore, to better match the fields of the laser radiation and the electron wiggler emission in the far-field region, the laser frequency must be red shifted by the same amount. Furthermore, the transverse mode of the laser beam must match the transverse mode of the spontaneous emission from an electron passing through the wiggler, and the laser spectral bandwidth must match the spectrum of the electron wiggler emission.

The energy exchange $\Delta E$ can be calculated by considering the cross-field term in the total field energy $A_{\text {total }}=\iint\left|\vec{E}_{L}(\vec{r}, \omega)+\vec{E}_{S}(\vec{r}, \omega)\right|^{2} d \vec{r} d \omega$, where $\omega$ is the frequency and $\vec{r}$ stands for all spatial coordinates. This term shows a superposition of the laser field $E_{L}$ and the spontaneous emission field from a single electron passing through the wiggler $E_{S}$ [Zhol1, Scho1]:

$$
\Delta E=2\left(A_{L} A_{W} \frac{M_{W} / \xi}{M_{L}} \eta_{e m i t}\right)^{1 / 2} \cos \phi \text { valid for } M_{W} / \xi \leq M_{L},
$$


where $A_{L}$ is the laser pulse energy, $M_{W}$ is the number of wiggler periods, $M_{L}$ is the number of optical cycles in the FWHM laser pulse length, and $A_{W} \cong 2.5 \alpha \hbar \omega_{L}$ is the energy spontaneously radiated by a single electron passing through the wiggler with $K>>1$ [Alfe, Zhol2], $\alpha$ is the fine structure constant, $\hbar$ is the Plank's constant, and $\omega_{L}=2 \pi c / \lambda_{L}$. Since each electron slips through $M_{W}$ optical cycles as it propagates through the wiggler, increasing the laser pulse length beyond a number of optical cycles of $M_{W} / \xi$ does not increase the energy exchange. The constant factor $\xi \approx 1.4$ results from matching the spectrum of a laser pulse (assumed to be Gaussian) to the spontaneous emission spectrum from the wiggler described by a sinc function, which is a Fourier transform of a square pulse of $M_{W}$ optical cycles in time. The non-zero size and divergence of the electron beam contributes to some loss in the amplitude of the energy modulation integrated over the transverse distribution of electrons. This is accounted for in Eq. (2) by the coefficient $\eta_{e m i t} \approx w_{0}^{2} /\left(w_{0}^{2}+2 \sigma_{x-e}^{2}\right)$, where $\sigma_{x-e}$ is the rms size of the electron beam, and $w_{o}$ is the waist size of the laser beam [Scho2].

Alternatively, one can also calculate $\Delta E$ by evaluating the integral $\Delta E=\int E_{L}(\vec{r}, t) v_{x}(\vec{r}, t) d t$, where $v_{x}$ is the electron velocity along the electron trajectory in the wiggler [Morp]. The equivalence of both approaches is demonstrated in [Zhol2]. We note that the former method affords calculations in analytical form even when the wiggler is detuned very far from the resonance condition (see Fig. 2), while it is difficult with the later method. 


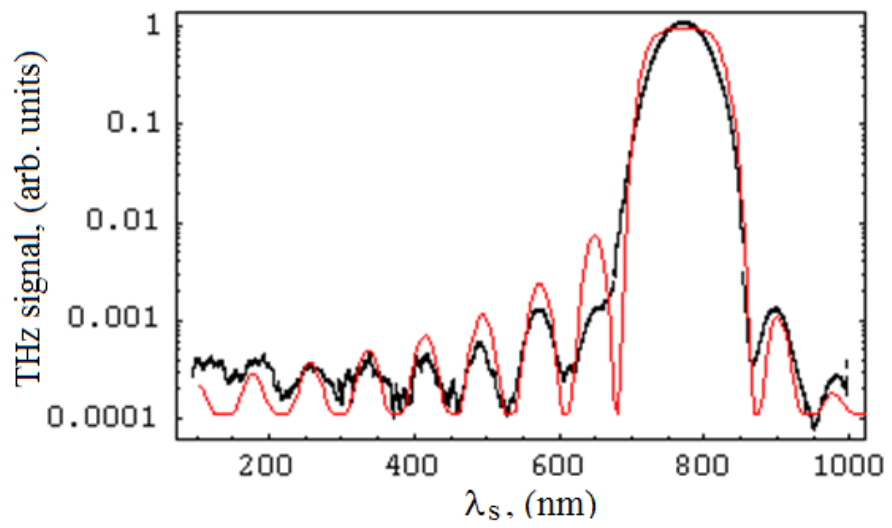

Figure 2. THz signal produced by the electron bunch experienced interaction with the laser as a function of various wiggler gaps corresponding to a wide range of $\lambda_{S}$ [Zhol2]. The black curve shows the measurement, and the red curve shows the calculated value for the integral over $\omega$ for the product of $E_{L}(\omega)$ and $E_{S}(\omega)$. Both curves are normalized to a value at the peak at $\lambda_{s}=\lambda_{L}=780 \mathrm{~nm}$. The characteristic oscillations in the signal are due to oscillating feature of a sinc function. Precise connection of the THz signal shown here to energy modulation of electrons is discussed later.

Figure 3 shows the amplitude of the energy modulation versus laser pulse energy measured at the BESSY-II light source in comparison with a theoretical prediction [Khan]. Approximately two times larger laser pulse energy was used in the experiment to obtain the theoretically predicted energy modulation as indicated by arrow in Fig.3. A similar observation was reported in [Scho3] for an experiment conducted at the ALS. No certain explanation for this discrepancy had been found except an assumption of possible phase-front distortions in the amplified laser pulses. 


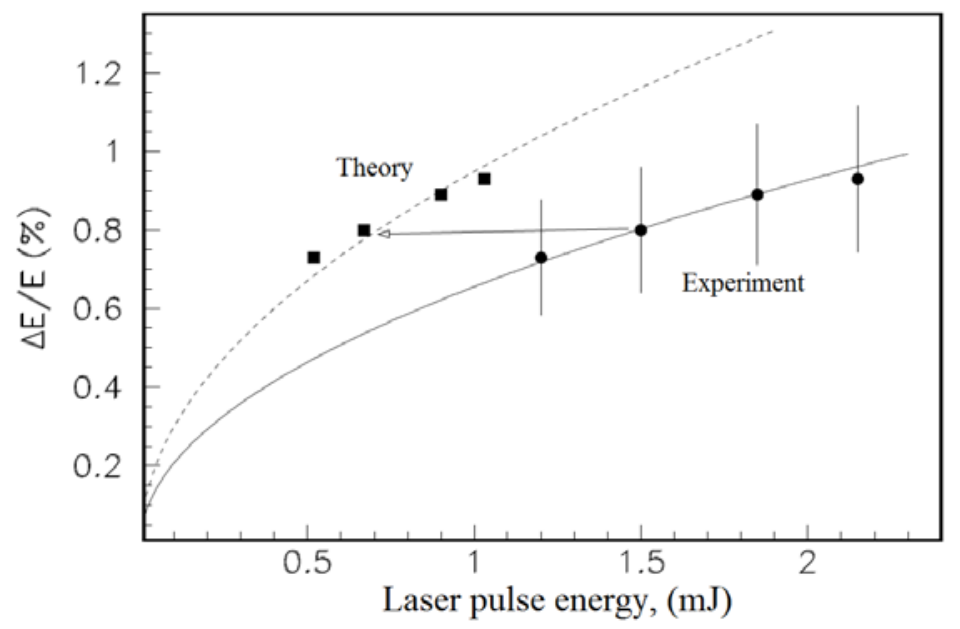

Figure 3. Energy modulation amplitude versus laser pulse energy. Dots are the measured values and squares are the expected values.

As the laser pulse co-propagates with the electron bunch through the wiggler, it experiences gain that is equivalent to the single-pass gain of a FEL [Elia, Morh]. Measurements of the spectral dependence of the FEL gain provide an effective diagnostic of the efficiency of the interaction between the laser pulse and the electron bunch. This is so because reaching the optimum gain requires the same mode-matching conditions required for optimum energy exchange between the laser pulse and the electron bunch [Made1, Made2]. Furthermore, the small-signal gain is independent of the laser pulse energy and, thus, can be measured with unamplified laser pulses from the laser oscillator. Conveniently, one can use a pair of interference filters that select the portion of the light spectrum where the gain is at maximum and where the gain is at minimum (see Fig. 4) after the laser interaction with the electrons. This allows differential detection, which effectively doubles the measured gain signal and eliminates noise due to laser-pulse energy fluctuations. Figure 5 shows the measured laser gain and a comparison with the predicted gain (following [Amir, Scho2] ): 


$$
\operatorname{gain}(v)=-2 \eta_{\text {emit }} \frac{K^{2} / 2}{1+K^{2} / 2} \frac{\left(2 \pi M_{W}\right)^{2}}{\gamma} \frac{I_{\text {peak }}}{I_{A}} q \frac{\partial}{\partial v}\left[\int_{-1 / 2}^{1 / 2} d \tau \frac{\cos \left[v \tau-\theta_{G}\right]}{\left(1+q^{2} \tau^{2}\right)^{1 / 2}}\right]^{2},
$$

where $I_{\text {peak }}$ is the measured peak bunch current, $I_{A}=e c / r_{e} \cong 17 \mathrm{kA}$ is the Alfvén current $\left(r_{e}\right.$ is the classical electron radius), $q=L_{W} / Z_{R}$, where $L_{W}$ is the length of the wiggler, $Z_{R}$ is the Rayleigh length of the focused laser beam, and $v=2 \pi M_{W}\left(\lambda_{L}-\lambda_{S}\right) / \lambda_{L}$ is the detuning. The phase term $\theta_{G}=\arctan (q \tau)$ is the Guoy phase advance through the laser focus. Figure 5a shows the measured laser gain as a function of time delay between the laser pulse and the electron bunch. This laser/e-beam cross-correlation by itself is an accurate measure of the electron bunch temporal shape, with a resolution determined by the timing jitter ( 1 ps) in the synchronization system. The maximum gain occurs approximately for

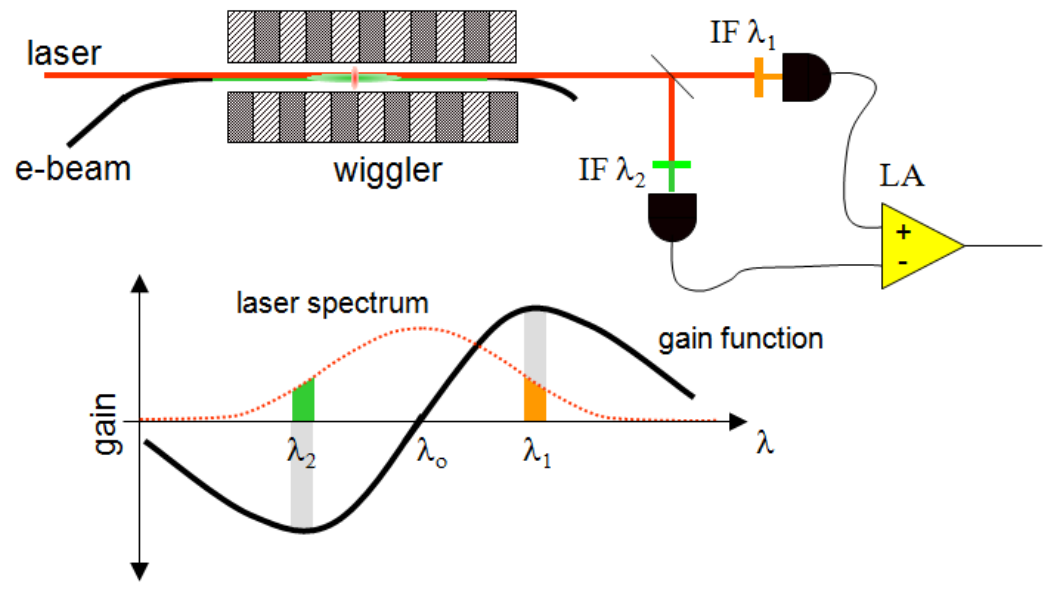

Figure 4. A schematic of the gain measurement (IF-interference filter, LA-lock-in amplifier). 

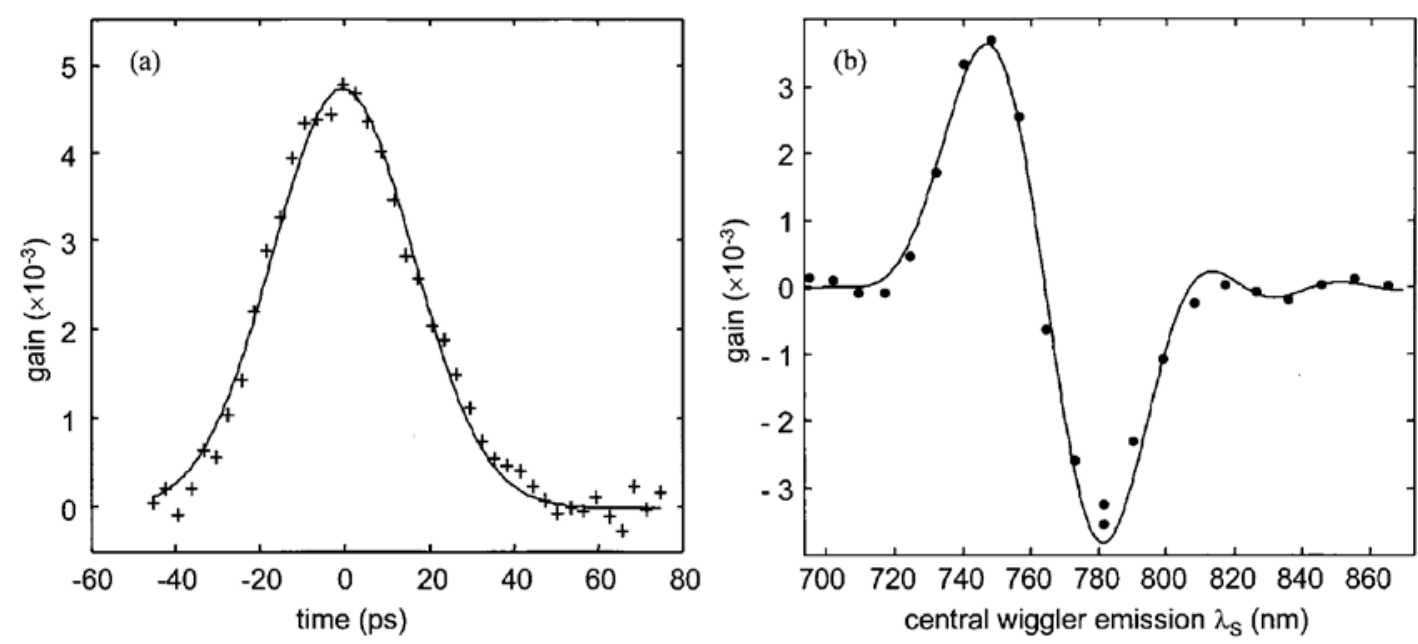

Figure 5. (a) A low signal gain as a function of delay between the laser pulse and the electron bunch (at optimum wiggler gap). Solid line is a Gaussian fit with $\sigma_{\mathrm{z}}=16.6 \mathrm{ps}$. (b) Spectral dependence of the gain as a function of wiggler emission wavelength (adjusted via the wiggler gap). Solid line is the gain predicted from Eq. (3) with $I_{\text {peak }}=23 \mathrm{~A}$, dots are the measured values.

$Z_{R} \approx L_{W} / 4$, and this determines the transverse laser mode necessary to achieve optimum interaction with the electron beam with the waist size in the wiggler center $w_{0}=\sqrt{Z_{R} \lambda_{L} / \pi}$. (We notice that in the case of the flat electron beam cross section, one can employ a tighter focusing of the laser in the vertical plane to achieve a stronger energy modulation of electrons using the same laser pulse energy.) Because of the correspondence between the low signal FEL gain and the energy exchange (gain $\Delta E^{2}$ ) [Made2], the good agreement between the predicted and measured gain indicates that the maximum possible energy exchange $\Delta E$ can be achieved with a high-quality laser beam.

The energy modulation can be exploited in several ways to generate sub-ps $\mathrm{x}$-ray pulses with minimal background contribution from the remaining electrons in the long bunch. One approach is to generate x-rays from a dispersive region of the storage ring, i.e., a region where the transverse beam size is determined primarily by the electron energy spread. Then an aperture can be used at an image plane of the source (created by 
the beamline optics) to select $\mathrm{x}$-rays originating only from the transversely displaced femtosecond electron slice (see Fig. 1c). Any long-pulse background will be determined by the tails in the transverse distribution of the unmodulated electrons. One can use the natural horizontal dispersion or create a vertical dispersion bump in a bend magnet or an undulator [Stei] and take advantage of the smaller vertical beam size. Knife-edge measurements of the electron-beam profile at the Advanced Light Source (ALS) indicate that it follows a Gaussian horizontal distribution out to $5 \sigma_{x-e}$. The sub-ps x-ray pulses can also be generated in an undulator and isolated by using a high-resolution monochromator to take advantage of the fact that the sub-ps electron slice will generate $\mathrm{x}$-rays that are correspondingly shifted in energy. In this case, the long-pulse background will be determined by the spectral resolution of the undulator and monochromator. In either approach, care must be taken to reduce any non-specular scattering from the x-ray optics, which will contribute to the long-pulse background by mixing x-rays originating from different transverse coordinates, or by mixing x-rays of different energies. A nonspecular background of $5 \times 10^{-4}$ has been measured in the image plane at a position of $5 \sigma_{x-e}$ along the horizontal direction following a single vertical reflection at grazing incidence from a toroidal imaging optic using x-rays at $2.4 \AA$ [Chur]. The sub-ps x-rays from an undulator can also be isolated by creating angular dispersion in the electron beam as shown in Fig. 6. Because no imaging optics is placed in front of the mask, an excellent signal-to-background ratio was obtained in this case shown in Fig. 7 [Khan]. 


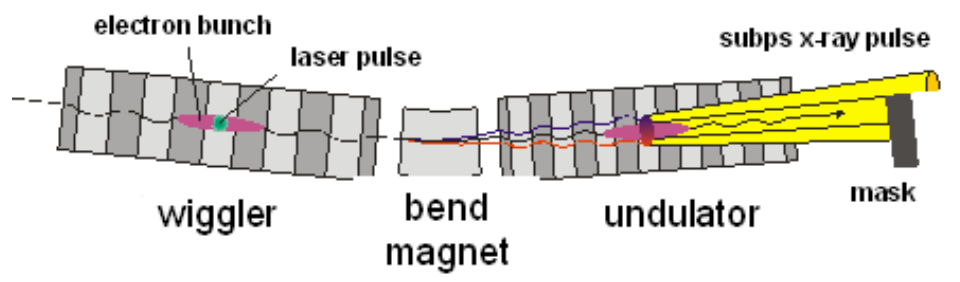

Figure 6. A schematic of angular separation of the sub-ps x-ray pulses emitted in the undulator with the mask blocking the radiation of core electrons at the undulator exit. Courtesy S.Khan.

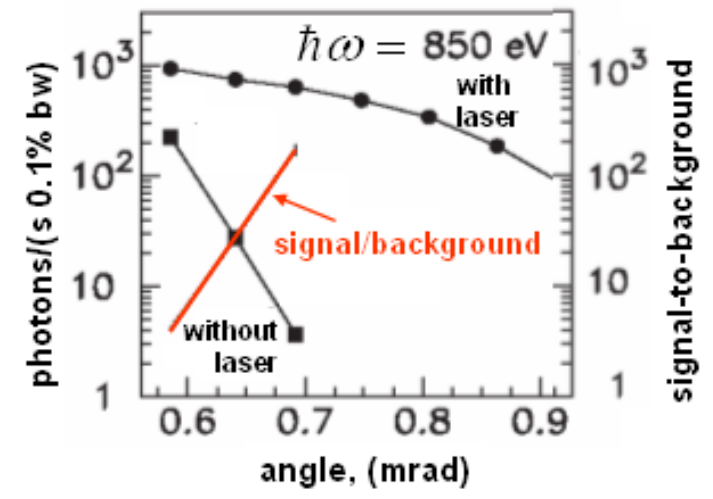

Figure 7. Detected photon rate per $0.1 \%$ bandwidth versus cutoff angle with (dots) and without (squares) laser. Red line shows signal-to-background ratio. Courtesy S. Khan.

Following interaction with a femtosecond optical pulse in the wiggler, the temporal and spatial distributions of electrons within the bunch are determined by the characteristics of the storage ring lattice. At the entrance of the wiggler, the initial Gaussian electron distribution is described by

$$
P_{o}\left(x_{o}, x_{o}{ }^{\prime}, E_{o}, t_{o}\right) \sim \exp \left[-\frac{x_{o}{ }^{2}+x_{o}{ }^{2}+E_{o}{ }^{2}}{2}-\frac{t_{o}{ }^{2}}{2 \sigma_{t-e}{ }^{2}}\right],
$$

where $x_{o}, x_{o}{ }^{\prime}$, and $E_{o}$ are the horizontal beam size, divergence, and energy, normalized to their respective rms values $\left(\sigma_{x}, \sigma_{x^{\prime}}\right.$, and $\left.\sigma_{E}\right)$, and $\sigma_{t-e}$ is the rms bunch duration. Energy 
modulation of the electrons co-propagating with the laser pulse through the wiggler is described by

$$
E_{1}=E_{o}+\exp \left[-t_{o}^{2} / 4 \sigma_{t-L}^{2}\right] \Delta E(\phi) / \sigma_{E},
$$

where $\Delta E(\phi)$ is from Eq. (1) with $\phi=\omega_{L} t_{o}$, and $\sigma_{t-L}$ is the rms duration of the laser field envelope. The additional factor of 2 in the denominator of the exponent accounts for the phase slippage between each electron and the laser field. Note that if the spontaneous emission spectrum is approximately matched by a Gaussian laser pulse spectrum, then the phase slippage is accounted for by using effective laser pulse duration of $\sim \sqrt{2} \sigma_{t-L}$. The electron distribution at the radiating source is given by

$$
P_{R}\left(x_{R}, t_{R}\right)=\iint d E_{1} d x_{0}^{\prime} P_{o}\left(x_{R}, x_{0}^{\prime}, E_{1}, t_{R}\right),
$$

where $x_{R}=x_{o} \cos \mu_{x}+x_{0}^{\prime} \sin \mu_{x}+\eta \cdot E_{1}, \quad \mu_{x}$ is the betatron phase advance between the interaction point and the radiation source, and $\eta$ is the ratio of the dispersive beam size to the betatron beam size at the radiation source. The transformation $t_{R}=t_{o}+\tau_{E} E_{1}+\tau_{x} x_{O}+\tau_{x^{\prime}} x_{0}^{\prime}$ accounts for the electron path length differences (time delays) due to the electron offset from the equilibrium with the rms values in the coordinate $\tau_{x}$, angle $\tau_{x^{\prime}}$, and energy $\tau_{E}$.

Figure 8 shows a calculated electron distribution of a laser-modulated bunch following propagation through one and one-half arc sectors of the ALS storage ring (as shown in Fig. 1) from the interaction point in the wiggler to after the high-dispersion (bend) region. Note that one arc sector is from the middle of one straight section to the middle of the following straight section. 


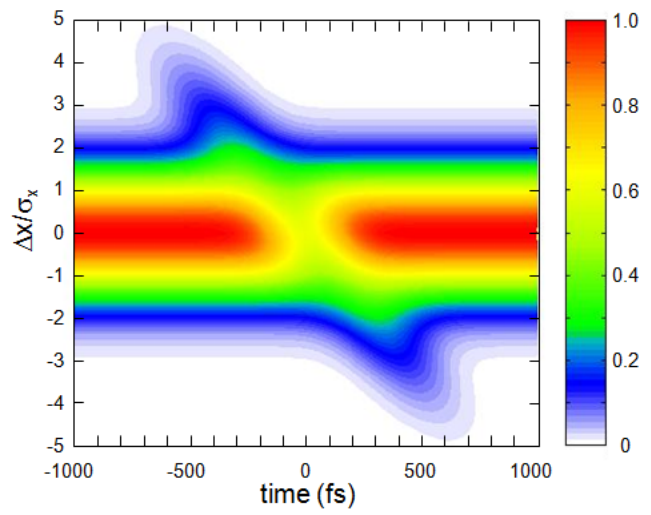

Figure 8. A calculated 2D electron density distribution (as a function of time and horizontal displacement normalized on the rms size) after electron bunch propagation through 1.5 arc sectors at the ALS from the wiggler to the bend magnet.

The sub-ps slice of electrons modulated by the laser is clearly separated from the main electron bunch since electrons with $\Delta E>0$ follow a different trajectory in this dispersive region than do the electrons with $\Delta E<0$. Furthermore, due to path-length differences (caused by time-of-flight properties of the storage ring), electrons with $\Delta E<0$ accumulate toward the head of the bunch while electrons with $\Delta E>0$ accumulate toward the tail of the bunch, giving rise to the time-skew observed in the electron distribution. The same time-of-flight properties also cause a temporal smearing of the bunch distribution due to the nominal rms electron energy spread $\sigma_{E}$, as well as a hole or dark pulse in the central core of the electron bunch. This time structure can be directly exploited to generate ultrashort synchrotron x-ray pulses with a duration approximated by

$$
\sigma_{x-r a y}^{2} \cong 2 \sigma_{t-L}^{2}+\tau_{E}^{2}+\tau_{x}^{2}+\tau_{x^{\prime}}^{2}
$$

where the factor of 2 accounts for the phase slippage between the laser pulse and each electron as they traverse the wiggler (assuming the wiggler spontaneous emission and laser pulse spectra are appropriately matched). 
Direct measurement of sub-ps x-ray pulses is difficult, and reliable methods have yet to be developed. Since the time structure of the electron bunch spontaneous emission is invariant over the entire spectrum of the spontaneous emission, one can use visible synchrotron radiation ( 2-eV photon energy) from a bend magnet instead of from the $\mathrm{x}$ rays to measure the temporal and spatial distribution of the synchrotron emission using optical cross-correlation techniques. In the experiment at the ALS, a visible light from a bend magnet was collected and imaged onto a slit. This allowed for the selection of specific horizontal regions of the synchrotron radiation originating from corresponding horizontal regions of the electron beam source (as illustrated in Fig. 1). Following the slit, the visible synchrotron light was focused onto a BBO crystal along with a delayed pulse from the laser system. The sum-frequency photons $(\sim 3.5-\mathrm{eV}$ photon energy) were counted as a function of the relative time delay between the first laser pulse, which was used to modulate the electron energy, and the second pulse, which was used for crosscorrelation measurements. (Note that during these measurements the first laser pulse remains fixed and coincident in time with the electron bunch in the wiggler.) Figure 9 shows the results of measurements obtained with two slit positions. The measured pulse duration is $\sim 150 \mathrm{fs}$.
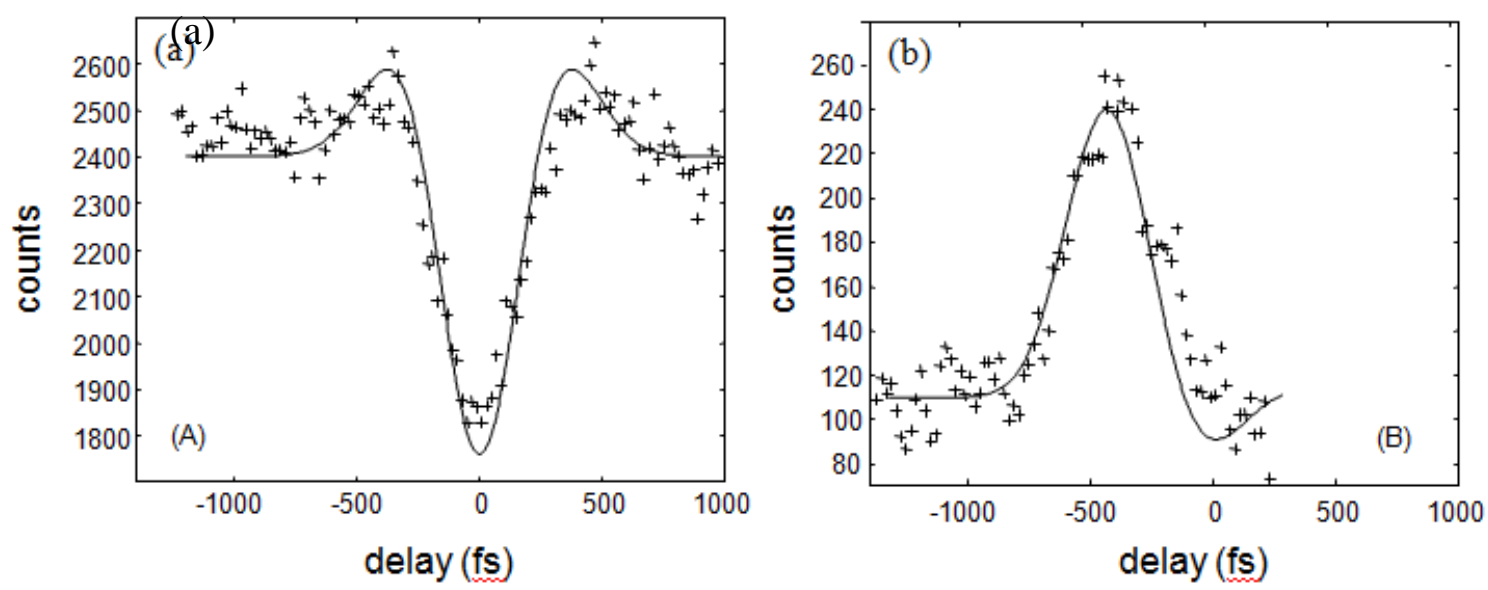
Figure 9. Cross-correlation measurements between a delayed laser pulse and synchrotron radiation originating from an energy-modulated electron bunch. (a) Synchrotron radiation from the central core: $\pm 3 \sigma_{x-e}$ of the electron bunch is selected revealing the sub-ps hole or dark pulse. (b) Synchrotron radiation from the horizontal wings: $+3 \sigma_{x-e}$ to $+8 \sigma_{x-e}$ of the electron bunch is selected. Solid lines are from a model calculation of the spatial and temporal distribution of the energy-modulated electron bunch following propagation through 1.5 arc sectors at the ALS using the storage ring parameters $\tau_{E}=103 \mathrm{fs}, \tau_{x}=20 \mathrm{fs}$, $\tau_{\chi^{\prime}}=7 \mathrm{fs}$, and $\sigma_{E}=1.2 \mathrm{MeV}$. The measured laser pulse parameters are $A_{\mathrm{L}}=0.4 \mathrm{~mJ}$ and $\sigma_{\mathrm{t}-\mathrm{L}}$ $=60 \mathrm{fs}$.

The average flux, brightness, and spectral characteristics of the femtosecond x-ray pulses is determined from the nominal characteristics of the radiating bend magnet or insertion device scaled by three factors: $\eta_{1}=\sigma_{t-L} / \sigma_{t-e}, \eta_{2}=f_{L} / f_{B}$, and $\eta_{3} \approx 0.2$, where $f_{L}$ and $f_{B}$ are the laser and electron-bunch repetition rates, and $\eta_{3}$ accounts for the fraction of electrons that are in the proper phase of the laser pulse to get the maximum energy exchange suitable for creating the large transverse separation. Increasing the laser repetition rate provides the greatest opportunity to maximize the sub-ps x-ray flux. The practical limit is determined by the synchrotron radiation damping, which provides for recovery of the electron beam between interactions. By arranging the timing such that the laser interacts sequentially with each bunch in the storage ring, the time interval between interactions is given by $N_{B} / f_{L}$, where $N_{B}$ is the number of bunches in the ring. Furthermore, since the bunch slice is only a small fraction of the total bunch, an interaction interval corresponding to $30 \%$ of the storage ring damping time (e.g., of the order of a few ms) is sufficient to allow recovery of the electron beam between laser interactions.

In addition to sub-ps x-rays, the time structure of the energy-modulated electron bunch shown in Fig. 8 gives rise to synchrotron emission in the THz part of the spectrum, which is longitudinally and spatially coherent. Because of the strong signal of coherent 
emission, it was adopted at ALS [Byrd1], BESSY [Holl], and SLS [Schl] for an initial optimization of the laser-electron beam interaction and for feedback control of spatial and timing drifts between laser and electron beams during data logging in experiments with sub-ps x-ray pulses.

Sub-ps time-dependent phenomena are typically studied with pump-probe techniques in which the dynamic processes are initiated by a femtosecond laser or laserdriven ultrafast source and then probed, after a time delay, with a sub-ps x-ray pulse. Because the femtosecond laser initiates the sub-ps x-ray pulse, a precise time delay can be produced between pump and probe pulses. The time resolution is then limited by the overlap of the pump and probe pulses.

At the time of this writing, the slicing technique had been implemented at the ALS [Scho3], BESSY [Khan], and SLS [Schl], and studies are underway for its implementation at SOLEIL [Nadj].

\subsection{RF orbit deflection}

Another way to force an ultrashort slice of the electron bunch to emit photons in a different direction than other electrons was proposed in [Zhol3] and independently in [Kato]. The idea is illustrated in Fig. 10. 


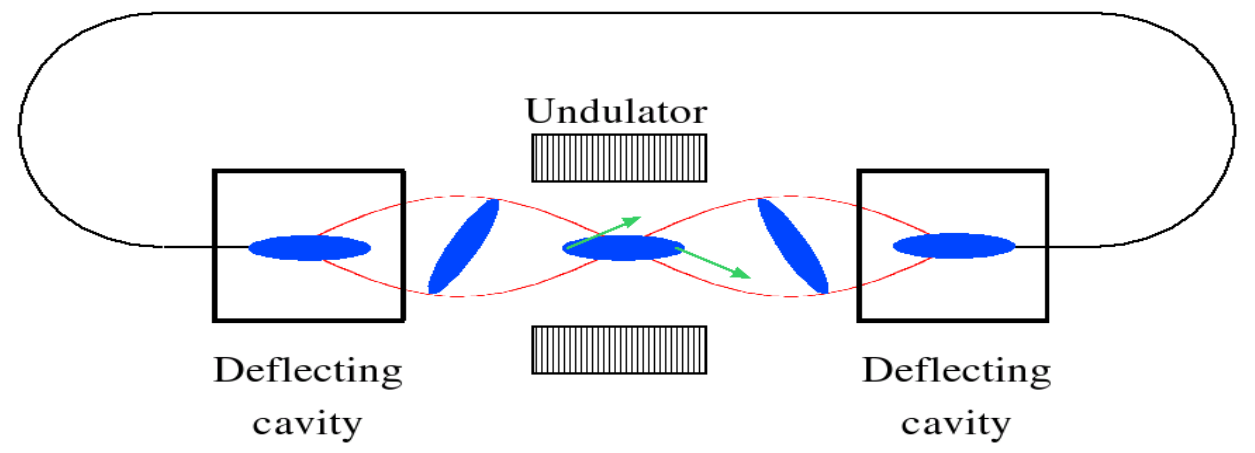

Figure 10. A schematic of an rf orbit deflection technique for generation of sub-ps xray pulses. Blue ellipses show side views of the electron bunch at several locations. Green arrows show intended directions for the electron bunch head and tail.

An electron bunch passes a deflecting cavity where electrons receive a timedependent kick in the vertical direction. This kick forces head electrons to move in one direction and tail electrons in the opposite direction. Thus, a quarter of the betatron wave downstream of the electron bunch develops a large tilt in the vertical plane. This tilt disappears half a betatron wave downstream, but the head and tail electrons keep moving in different directions. In the undulator, such an electron bunch emits an x-ray pulse with a time-dependent chirp that can be observed if the angle between the head and tail trajectories is larger than the diffraction-limited angle of the undulator radiation $\sigma_{r^{\prime}}=\sqrt{\lambda_{x} / \pi L_{u}}$ (here $\lambda_{x}$ is the x-ray wavelength and $L_{u}$ is the undulator length) and an rms angular spread of electrons $\sigma_{y^{\prime}}$. In fact, if the angle between the head and tail trajectories is sufficiently large, the radiation fan can be sliced into many diffractionlimited x-ray beams that can be spatially separated. The second deflecting cavity located another half betatron wave downstream cancels the initial kick. Placing the aperture in the beamline and selecting emission coming from only a single $\mathrm{x}$-ray slice allows one to obtain a short x-ray pulse 


$$
\sigma_{\tau-\text { xray }} \approx \frac{E_{b}}{e U} \frac{1}{2 \pi f_{r f}} \sqrt{\frac{\varepsilon_{y}}{\beta_{r f}}\left(1+\frac{\sigma_{r^{\prime}}^{2}}{\sigma_{y^{\prime}}^{2}}\right)},
$$

where $U$ is the "deflecting voltage", e.g., the integral of the electric field taken along the line with a vertical offset where the rf field has a maximum amplitude, $f_{\mathrm{rf}}$ is the deflecting cavity frequency, $\varepsilon_{y}$ is the vertical electron beam emittance, and $\beta_{r f}$ is the vertical beta function in the deflecting cavity. Figure 11 shows a predicted pulse length for APS storage ring (a) as a function of the deflecting voltage, and (b) as function of the photon energy for $e U=4 \mathrm{MeV}$ [Borl1]. Other parameters used in the calculations are: $f_{\mathrm{rf}}=2.8 \mathrm{GHz}, \beta_{\mathrm{rf}}=20 \mathrm{~m}, \varepsilon_{\mathrm{y}}=4 \times 10^{-11} \mathrm{~m}$, and $E_{\mathrm{b}}=7 \mathrm{GeV}$. As follows from Eq. (8), when $\sigma_{r^{\prime}}>\sigma_{y^{\prime}}$, the pulse duration is dominated by diffraction. This happens at relatively low photon energies. At high photon energies the pulse duration is independent of the x-ray wavelength and is dominated by angular divergence of electrons defined by the vertical emittance.
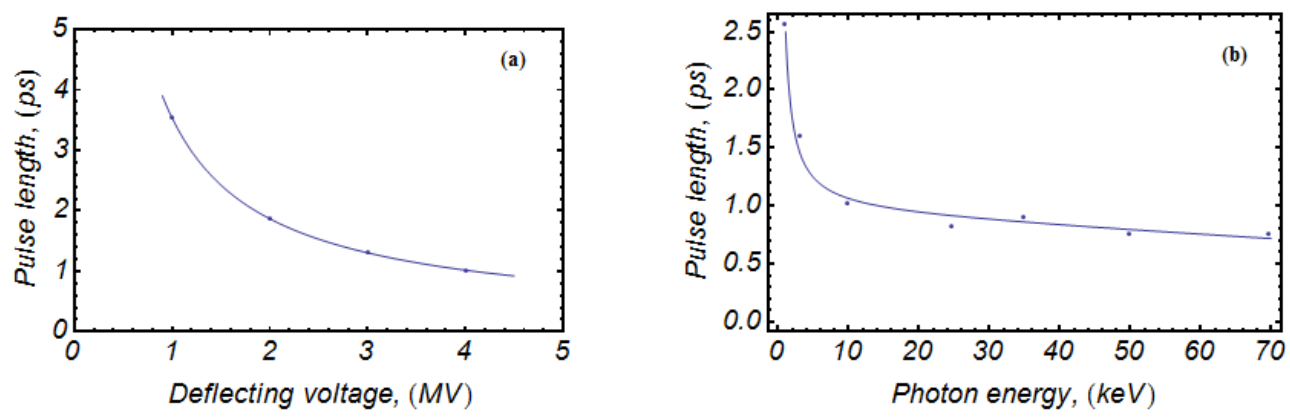

Figure 11. Predicted x-ray pulse length measured at approximately $\pm \sigma$ level of intensity distribution shown (a) as a function of deflecting voltage for $10-\mathrm{keV}$ photon energy and (b) as a function of the photon energy. The data is taken from a design study for the APS upgrade [Borl1]. 
The aperture cuts the x-ray flux and a predicted tradeoff between desirable pulse duration and transmission efficiency is shown in Fig. 12. Shorter pulses can be obtained at smaller transmission and with larger deflecting voltage.

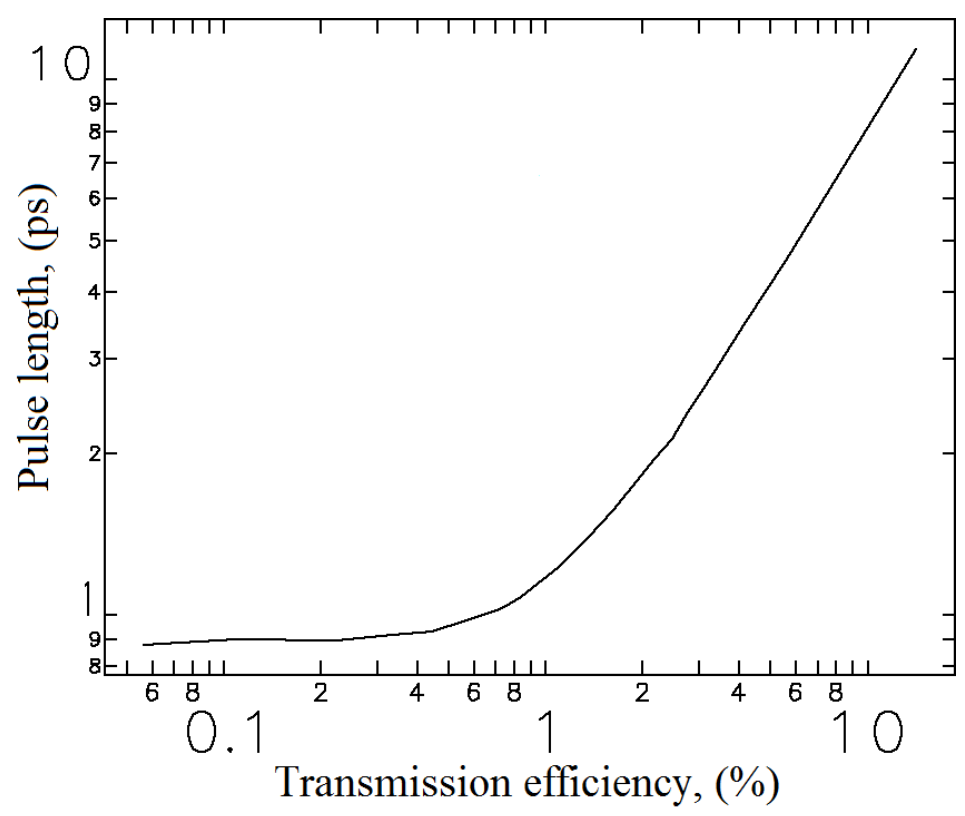

Figure 12. Predicted $\mathrm{x}$-ray pulse length measured at approximately $\pm \sigma$ level of intensity distribution as a function of the transmission efficiency of the $\mathrm{x}$-ray flux through the aperture calculated for APS using 4 MV deflecting voltage [Borl1].

A pair of asymmetrically cut crystals can be used in the x-ray beamline in order to collect the entire emission of the electron bunch without compromising the $\mathrm{x}$-ray pulse duration [Zhol3]. This crystal has different incident and diffractive angles (see Fig. 13), that allow it to create a path length difference across the photon pulse to compensate the difference in the arrival time of photons emitted by different sections of the electron bunch. However, a potential $30 \%$ increase in transmission comes at the expense of a more complex x-ray beamline that must accommodate a large beam size. 


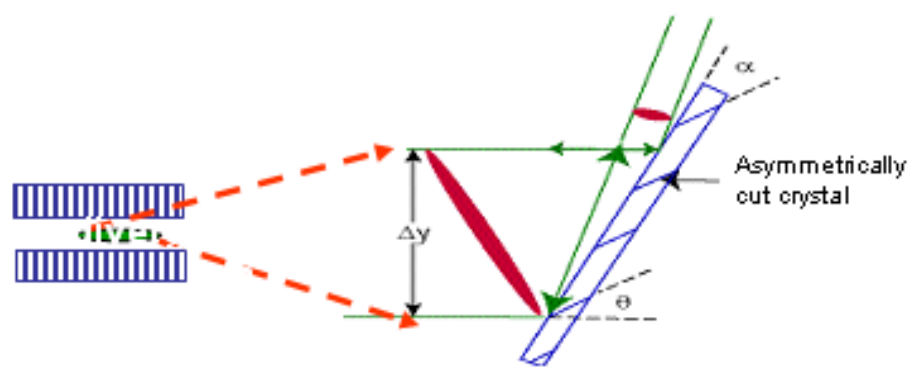

Figure 13. An illustration of an x-ray pulse compression using an asymmetrically cut crystal.

A comprehensive study of the rf deflection technique for APS [Borl2] revealed a large blow-up of the vertical emittance that compromised the very basics of this method for obtaining short x-ray pulses. Errors affecting the vertical emittance are unbalanced cavity voltages, errors in the beta functions, errors in the betatron phase advance between the cavities, cavity rolls, and rolls of magnets between the cavities. It was found that cavity rolls have a weak effect. Beta function errors can be compensated by changing the relative voltage of the second cavity. Phase advance error can be compensated by changing the relative voltage of the first and second sets of cells of the second cavity. Magnet rolls can be corrected locally with additional skew quadrupoles. Then, it was found that a large blow-up of the vertical emittance was mainly caused by sextupole lenses used for chromaticity correction between cavities. The effect was attributed to coupling of the horizontal and vertical emittances caused by a vertical tilt in the electron bunch [Saja]. This tilt forced electrons to pass sextupoles with a vertical offset whose magnitude oscillated with the synchrotron frequency. Then a variant of the chromaticity correction using a new set of sextupole gradients was found where the coupling impact of one group of sextupoles was largely compensated by the coupling impact of the other group of sextupoles [Borl3]. 
A synchronization between laser pump and $\mathrm{x}$-ray probe pulses in this technique with a sup-ps jitter can be obtained by linking the laser pulse to a zero crossing phase of the deflecting cavity.

At the time of this writing, the rf orbit deflection technique had not been implemented at any of the existing storage ring light sources. Studies are underway for its installation at the APS, SPring8 [Fuji], and the Taiwan Photon Source [Ghas].

\section{Generation of Ultrashort X-ray Pulses using a Linear Accelerator}

\subsection{The Sub-Picosecond Pulse Source (SPPS)}

A linear accelerator (linac) is much better suited than a storage ring for the task of producing ultrashort x-ray pulses. Most of the subtle reasons that prevent a short bunch in a storage ring simply disappear when a linac is considered. The perfect example is the Sub-Picosecond Pulse Source (SPPS). This source was based on the SLAC linac and operated throughout construction of the Linac Coherent Light Source (LCLS). It was then superseded by the LCLS (see discussion in the FEL section).

Short electron bunches were produced in the linac in a three-stage bunch

compression process [Corn, Krej]. First, the electron bunch with approximately $2 \times 10^{10}$ electrons per bunch and 20-ps bunch length was extracted from the North Damping Ring (NDR) and compressed to approximately 4 ps in the Ring To Linac (RTL) beamline. Then a combination of specially installed chicane (at a location with $9-\mathrm{GeV}$ beam energy) and energy chirp developed by off-crest acceleration and wakefields was used to compress the electron bunch down further to 160 fs. The third stage of compression occurred in the bends of the Final Focus Test Beam line, which produced an 80-fs 
FWHM electron bunch at 28-GeV beam energy. In all the stages of compression the collective effects driven by a charge of the electron bunch and CSR had to be carefully treated [Emma1]. A 10-m-long undulator installed in the FFTB facility generated spontaneous radiation at the undulator fundamental photon energy of $8.3 \mathrm{keV}$. The inability to precisely control the arrival time of the electron bunch led to a significant reduction in temporal resolution in pump-probe experiments. To circumvent this problem a single-shot determination of the arrival time with respect to the laser pulse using electro-optics sampling was developed and successfully applied to yield better than 60-fs resolution [Cava, Lind2].

\subsection{Generation of Femtosecond X-rays via Relativistic Thomson Scattering}

Alternatively, when a high-energy linac is not available, the x-rays can be produced via scattering of laser light off a relativistic electron beam [Arut, Milb] (Thomson scattering). Although, this process has been known for a long time, it was not used for production of ultrashort $\mathrm{x}$-ray pulses until the clever idea of crossing laser and electron beams at a $90^{\circ}$ angle was proposed [Kim1]. In this geometry, shown in Fig. 14, the interaction interval (and therefore the x-ray pulse duration) is limited to the transit time of the laser pulse across the waist of the electron beam. 


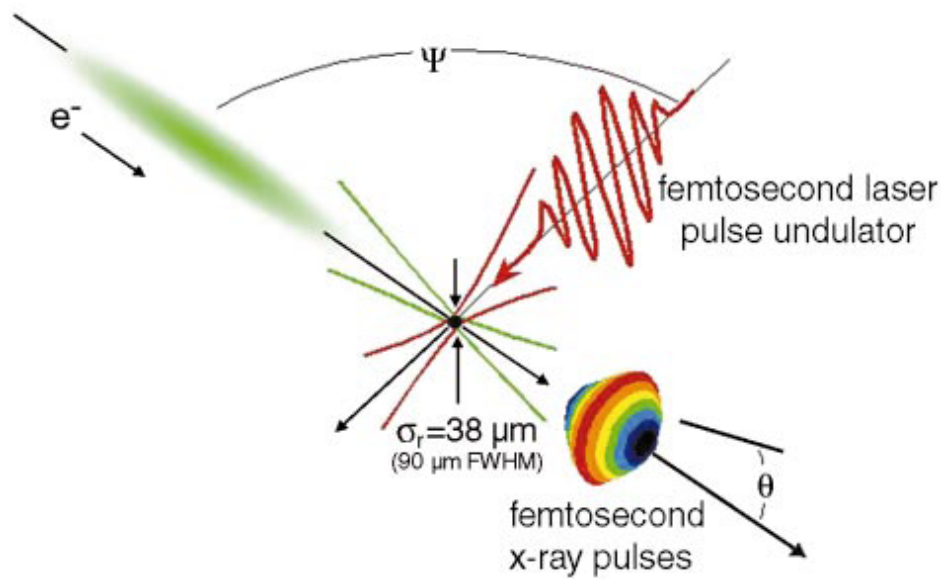

Figure 14. A schematic for generating sub-ps x-ray pulses via Thomson scattering.

At shallow scattering angles, the $\mathrm{x}$-ray pulse duration scales as $\psi$, however, the $\mathrm{x}$ ray yield also scales with the scattering angle. Thus $\psi=90^{\circ}$ is a compromise between $\mathrm{x}$ ray flux and pulse duration [Scho4, Leem].

The characteristics of sub-ps x-rays generated via Thomson scattering can be quantitatively described by considering the laser field as an electromagnetic wiggler for the electron beam. Since the wiggler period is the laser wavelength, short-wavelength photons can be generated from electron beams of moderate energy. In an analogy to Eq. (1), the $\mathrm{x}$-ray wavelength is given by

$$
\lambda_{x-\text { ray }}=\frac{\lambda_{L}}{2 \gamma^{2}} \frac{1+\gamma^{2} \theta^{2}+K_{L}^{2} / 2}{1-\cos \psi},
$$

where $K_{L}=e E_{L} \lambda_{L} / 2 \pi m c^{2}$ is the normalized vector potential of the peak laser field $E_{L}$. For typical laser intensities, $K_{L} \leq 1$. The x-ray flux (number of x-ray photons) in a narrow bandwidth $(\Delta \lambda / \lambda<<1)$ is given by [Kim1, Kim2]

$$
N_{x-\text { ray }} \cong \frac{\pi}{2} \alpha\left(\frac{K_{L}{ }^{2}}{1+K_{L}{ }^{2} / 2}\right) M_{L} \frac{\Delta \lambda}{\lambda} N_{e} \frac{\sigma_{t-L}}{\sigma_{t-e}} \text { for } \sigma_{t-L}<\sigma_{t-e} \text { and } \Delta \lambda / \lambda<<1 \text {, }
$$


where $M_{L}$ is the number of optical cycles in the laser pulse; $\sigma_{t-e}$ and $\sigma_{t-L}$ are the rms durations of the electron bunch and laser field envelope, respectively; and $N_{e}$ is the number of electrons in a single bunch.

A number of characteristics in the laser and electron beams have to be optimized in order to obtain the highest flux of x-ray photons with a given pulse duration. For example, the laser pulse energy can be increased, but for $K_{L} \geq 1$ the additional x-ray flux appears at higher harmonics and the source divergence increases $\sim K / \gamma[\mathrm{Kim} 2]$. Also, the interaction area (focal spot size) may be reduced provided that the electron beam emittance is also reduced to avoid increasing the rms divergence of the x-rays $\sigma_{\theta}$ determined by two factors [Kim2]:

$$
\sigma_{\theta}^{2} \cong\left[\frac{\varepsilon}{\gamma \sigma_{r}}\right]^{2}+\left[\frac{\lambda_{x-r a y}}{2 M_{L} \lambda_{L}}\right]
$$

The first term is due to to the non-zero electron beam emittance $\varepsilon$, which is normalized to $\gamma$, and the rms radius of the beam focus $\sigma_{r}$. The second term is due to diffraction from a finite-length source and is defined by $M_{L}$. Even for a zero-emittance beam this term will give rise to significant source divergence $\sigma_{\theta}=1.3$ to $4.2 \mathrm{mrad}$ calculated for the x-ray source in the 1- to $10-\mathrm{keV}$ photon energy range. The benefit of operating at longer laser wavelengths is cancelled by the corresponding reduction in $M_{L}$ (assuming fixed laser and x-ray pulse durations). The x-ray divergence can also be reduced by increasing the electron beam energy. However, since the wiggler period is an optical cycle, the x-ray energy rapidly exceeds the range of interest for many applications in studying structural dynamics in condensed matter. 
In the first demonstration of this technique, $\sim 300 \mathrm{fs} x$-rays at $30 \mathrm{keV}$ were generated using a laser pulse ( $\sigma_{t-L} \cong 60 \mathrm{fs}, \lambda_{\mathrm{L}}=0.8 \mu \mathrm{m}, 100 \mathrm{~mJ} / \mathrm{pulse}$ ) and a $50-\mathrm{MeV}$ electron bunch with $\sigma_{t-e} \sim 15$ ps focused to a spot size of $90 \mu \mathrm{m}$ FWHM [Scho4, Leem]. The same technique also had been applied for a production of ultrashort x-ray pulses in [Yang, Uesa].

The simplicity of the Thomson scattering approach is that the laser provides for slicing of the x-ray pulse duration via right-angle scattering and simultaneously acts as an undulator, thereby permitting in principle a precision synchronization for pump-probe experiments. On the other hand, we note that for the same average laser power, the slicing source described at the beginning of Part 1 provides several orders of magnitude higher average flux and brightness.

\section{Part 2: Coherent Emission from Free-Electron Lasers}

\section{Current enhanced self-amplified spontaneous emission and seeded FELs}

When x-ray free-electron lasers came into operation [Acke, Schr, Emma2], they changed the entire landscape for time-resolved studies with x-rays. Obtaining sub-ps xray pulses became routine and the current record remains at less than 10 fs FWHM [Ding1]. The number of photons per pulse went up by 6 to 8 orders of magnitude. Thus, the emphasis is now shifting to obtaining even shorter pulses as well as attaining adequate synchronization between the x-ray probe and the laser pump pulses. Although the incoherent $\mathrm{x}$-rays produced in SPPS experiments were relatively easy to time [Cava], the severe electron beam reshaping that occurs during the lasing process in the self- 
amplified spontaneous emission (SASE) FEL can in principle make upstream electron bunch measurements unreliable indicators of the $\mathrm{x}$-ray laser pulse duration and arrival time. The uncertainty can be removed by controlling the electrons, preferably with a laser that can also be used as a pump source, or provide a timing mark for a pump source. Possible implementation of this approach is shown in Fig. 15 (see also, [Zhol4, Zhol5]).

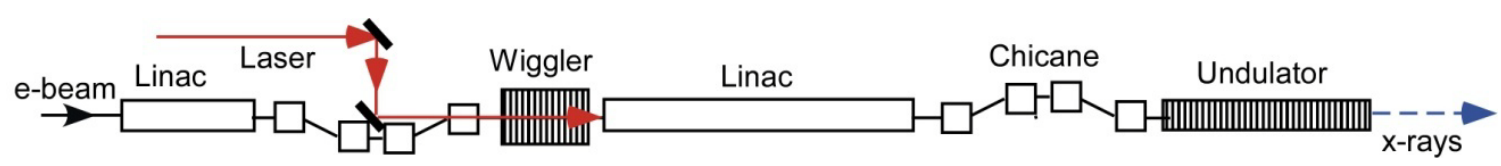

Figure 15. A schematic of a current enhanced SASE (ESASE) x-ray FEL.

In this scheme, the electron beam passes the linac and enters a wiggler magnet. At the same time a short laser pulse (shorter than the electron bunch minus a jitter in the arrival time of the electron bunch) enters the wiggler and co-propagates it with the electrons. The laser pulse overlaps only a short section of the electron bunch whose arrival time in the wiggler corresponds to the arrival time of the laser pulse. For convenience we call this section the working section (WS). Electrons in the WS interact with the laser field and emerge from the wiggler with an energy modulation. This is very similar to the slicing technique considered in Part 1, and all formulas given there for calculation of the energy modulation are applicable here. The laser pulse energy is chosen such that the amplitude of the energy modulation exceeds the uncorrelated energy spread of the electrons by a factor of 5 to 10 . Next, the electron beam enters a second linear accelerator and gains energy to reach the final energy. This acceleration does not affect the energy modulation introduced in the wiggler and does not produce noticeable relative longitudinal motion of electrons because of the ultra-relativistic electron energies. Following acceleration, the electron beam passes through a dispersive magnetic chicane 
that produces micro-bunching of the electrons in the WS and periodic enhancement of the electron peak current. Finally, the electron beam passes through a long undulator where electrons inside the WS produce enhanced SASE because of the current enhancement. The x-ray radiation produced by electrons outside of the WS has significantly less intensity because of the longer gain length at a significantly lower peak current. Thus, there is precise synchronization between the output $\mathrm{x}$-ray pulse and the laser pulse since only electrons from the WS, i.e., from the region that experienced interaction with the laser, produce intense x-rays. This feature can be used in pump-probe experiments. Moreover, by changing the duration of the laser pulse and adjusting the number of active wiggler periods, one can regulate the length of the WS and therefore the duration of the x-ray output. It is beneficial to have the same laser producing two laser pulses: one for energy modulation of electrons and one as a pump source. However, due to practical constrains, one may need two different lasers that could even be located hundreds of meters apart. In that case, they should be synchronized with the smallest possible error; as reported in [KimJ, Wilc, Byrd2, Löhl], the current state of the art of the timing systems based on stabilized optical fiber links is an error better than $10 \mathrm{fs}$.

The idea for electron been manipulation in FELs using an external laser has its roots in the concept of seeded FELs [Cson]. Seeded FELs employing the process of highgain harmonic generation (HGHG) [Yu] use laser for energy modulation of electrons in the first undulator, convert it into density modulation using a magnetic chicane to obtain a relatively large microbunching of electrons at a high harmonic of the laser frequency, and produce amplified radiation in the downstream undulator tuned on the FEL resonance at harmonic frequency. Seed signal can also be obtained via high-order harmonic 
generation [Schn, Rund, Wang, Arpi] using all optical technique as demonstrated in [Lamb]. Echo-enabled harmonic generation (EEHG) [Stup, Xian1] is another seeding option that utilizes two consecutive laser modulations, each with an attached dispersion section, to achieve microbunching at an even higher harmonic with remarkable upfrequency conversion efficiency. After they are built, seeded FELs will naturally possess a precise synchronization between laser and x-ray pulses as they are linked to each other through laser electron beam interaction that eventually leads to generation of $\mathrm{x}$-rays. Similar to ESASE, changing the duration of the laser pulse will directly affect the duration of the x-ray output.

\section{Generation of attosecond $x$-ray pulses}

It is rather natural to consider using decreasingly shorter and shorter seed laser pulses for production of the ultrashort x-ray pulses either in ESASE or in seeded FEL until it comes to a pulse containing just two optical cycles, e.g., the shortest pulse with a millijoule-level pulse energy demonstrated at the time of writing [Sart, Brab]. Then, one can use a wiggler with just one or two periods and obtain an ultrashort slice of energymodulated electrons. The waveform of this energy modulation closely resembles the waveform of the laser electric field that can be manipulated using the technique of carrier-envelope phase (CEP) stabilization [Jone, Balt, Itat, IEEE] primarily developed for a generation of the attosecond x-ray pulses using high harmonics produced in the interaction of a few-cycle laser pulse with atomic electrons; see reviews in [Brab, Krau] and references therein. Thus, for example, one can obtain a cosine-like waveform of the electron energy when the peak of the electric field is at the maximum of the envelope or a 
sine-like waveform when a zero crossing of the electric field is at the maximum of the envelope (see Fig.16).
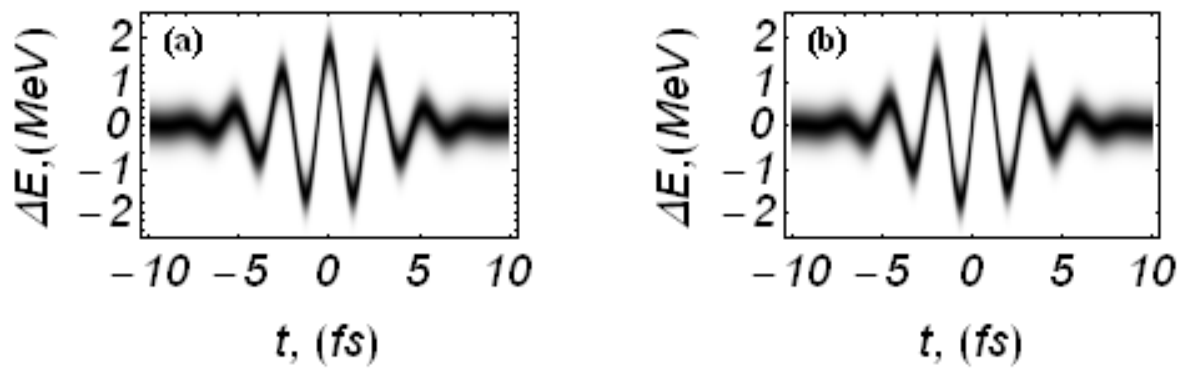

Figure 16. Density plot showing energy modulation of electrons produced in the interaction with a few-cycle, 800-nm-wavelength laser pulse with CEP stabilization interacting with the electron bunch in the wiggler magnet with two periods. Only a small fragment of the electron bunch longitudinal phase space cut at \pm 10 -fs points along the electron bunch is shown. (a) A cosine-like form, and (b) a sine-like form.

A unique opportunity offered by the waveform shown in Fig. 16 for a generation of the attosecond x-ray pulses in a free-electron laser was initially recognized in [Zhol6]. Since then many more ways of generating attosecond x-ray pulses using both waveforms had been found [Sald1, Sald2, Zhol7, Sald3, Ding2] as well as uses for these waveforms in combination with some additional techniques [Zhol8, Xian2, Zhol9]. Here we review just a few of them. In Fig. 17 we show a schematic of the technique that is most suitable for generation of soft x-ray pulses [Zhol6].

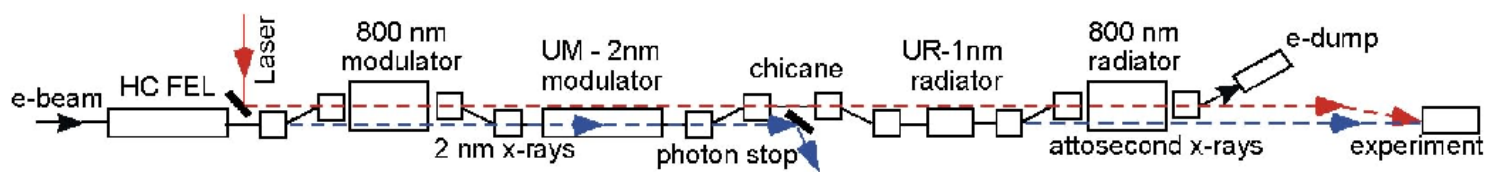

Figure 17. A schematic of the components involved in attosecond x-ray pulse production using a few cycle laser pulse with CEP stabilization and a soft x-ray FEL.

On the left is a source producing a coherent 2-nm-wavelength, 100-fs, 100-MW peak power x-ray pulse. While such sources do not exist today, studies of seeded FELs 
[Fawl1, Penn1, Penn2] have suggested approaches that are feasible in principle. As a specific example, the x-ray source at 2-nm wavelength is chosen to eventually produce 1nm wavelength attosecond radiation. The FEL can be configured such that only part of the electron bunch is used for x-ray generation, thus leaving another part near the bunch head whose instantaneous energy spread has not been degraded by previous FEL interaction in the upstream cascade. The existence of these "virgin" electrons can be ensured by an electron beam pulse duration sufficiently long ( $\geq 0.5 \mathrm{ps}$ ) to account for jitter in the arrival time of the electron bunch.

After exiting the FEL, an achromatic bend inserts the electrons into a two-period wiggler magnet “800-nm modulator.” Simultaneously, a few-cycle 5-fs-long laser pulse at 800 -nm wavelength and $~ 1-\mathrm{mJ}$ pulse energy enters this wiggler and co-propagates with the electrons. The relative timing between the arrival of the electron beam and the optical pulse is set such that the latter temporally overlaps the "virgin" electrons. We presume that the $\mathrm{x}$-ray HC FEL pulse will be seeded with a laser pulse that originates from the same laser source as the few-cycle laser pulse, which consequently permits tight synchronization between the two. Since the "virgin" ultra-relativistic electrons and the FEL x-ray pulse come from the same electron bunch, one can thus ensure temporal synchronization between each of these three beams.

The carrier-envelope phase of the few-cycle laser pulse is adjusted so that the peak electric field appears at the peak of the envelope when the laser pulse passes the wiggler center. The wiggler's magnetic period and undulator parameter $K$ are adjusted such that fundamental FEL resonance occurs at the laser wavelength $\lambda_{L}=800 \mathrm{~nm}$. The interaction with the laser light in the wiggler then produces a time-dependent electron 
energy modulation extended over a few optical cycles, as shown in Fig. 16(a). For the laser pulse parameters mentioned above, we expect central peak energy offset $\Delta \mathrm{E}_{0} \approx 15$ $\mathrm{MeV}$, which is a factor of 1.35 times larger than those of its two nearest neighbors. This relative difference is important when considering the 2-nm energy modulation to be induced in the following undulator.

A second isochronous bend after the wiggler magnet returns the electrons back to the original axis. The electrons now enter a long undulator-modulator (UM) (not shown to scale in Fig. 17) that serves as an energy modulator at 2-nm wavelength. The coherent, $\geq 100$-fs-long, 2-nm output pulse from the HC FEL co-propagates in the UM with electrons and arrives simultaneously with those electrons that experienced the strong energy modulation at $800 \mathrm{~nm}$. The undulator parameter $K$ of the $\mathrm{UM}$ is tuned such that only those electrons very near the peak of the 800-nm energy modulation have the correct energy for resonant FEL interaction with the 2-nm light. The other electrons fall outside the energy bandwidth of the UM and are not significantly modulated. The UM is shorter than one full FEL gain length, so there is little SASE action leading to unwanted microbunching at 2-nm wavelength throughout the 2-ps-long electron bunch.

Downstream of the UM the electrons enter a chicane with a time-of-flight parameter $R_{56}=750 \mathrm{~nm}$, which induces strong microbunching at $\lambda_{x}=2 \mathrm{~nm}$ x-ray wavelength and at higher harmonics $\lambda_{x} / \mathrm{n}$. Skipping the details of the mathematical analysis that can be found in [Zhol6], we plot in Fig.18 the bunching amplitude for $n=2$ (i.e., 1-nm wavelength) as theoretically predicted and as calculated by the GINGER simulation code [Fawl2]. 


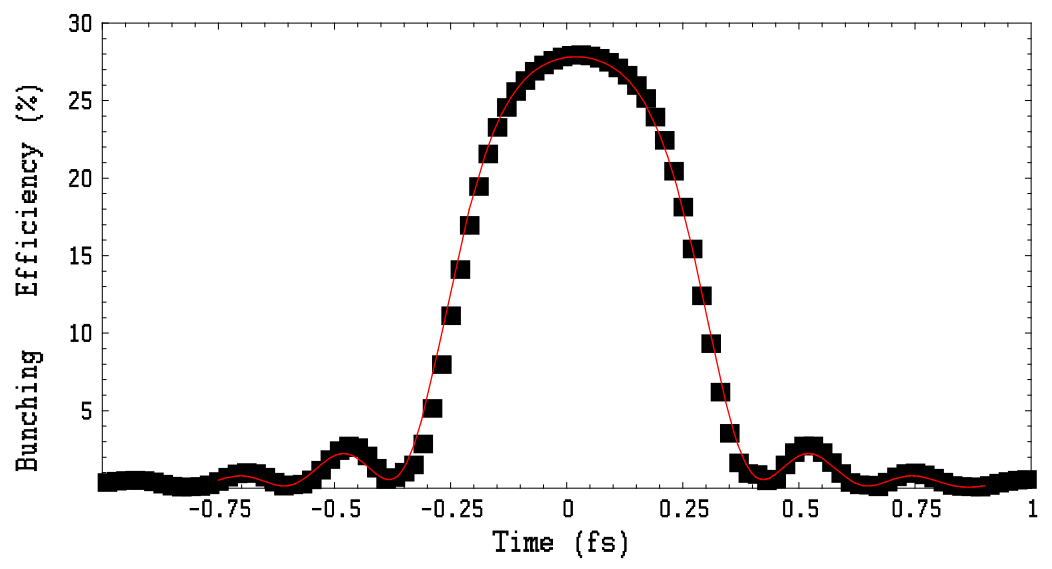

Figure 18. Bunching efficiency at 1-nm wavelength versus time along the electron bunch. The solid line shows analytical prediction and the dots are simulation results from GINGER. The FWHM of the peak is 530 attoseconds.

After the chicane, the electrons proceed to an undulator-radiator (UR) in microbunches and produce coherent emission at wavelength $\lambda_{x}=1 \mathrm{~nm}$. The interference of the waves emitted by all macroparticles defines the output envelope of the radiation field. The predicted radiation field intensity is shown in Fig. 19. There is a good agreement between the analytic predictions and the GINGER simulation. The rms width $\sigma_{t}$ of the peak is 110 -as FWHM for the radiator with a number of periods $N_{R}=80$ and 180 -as for the radiator with $N_{R}=45$. This is several times shorter than the bunching width structure shown in Fig. 3. This reduction is attributed to a destructive interference (due to temporal variation of bunching phase) occurring between waves emitted by microbunches on opposite sides of the bunching peak. 


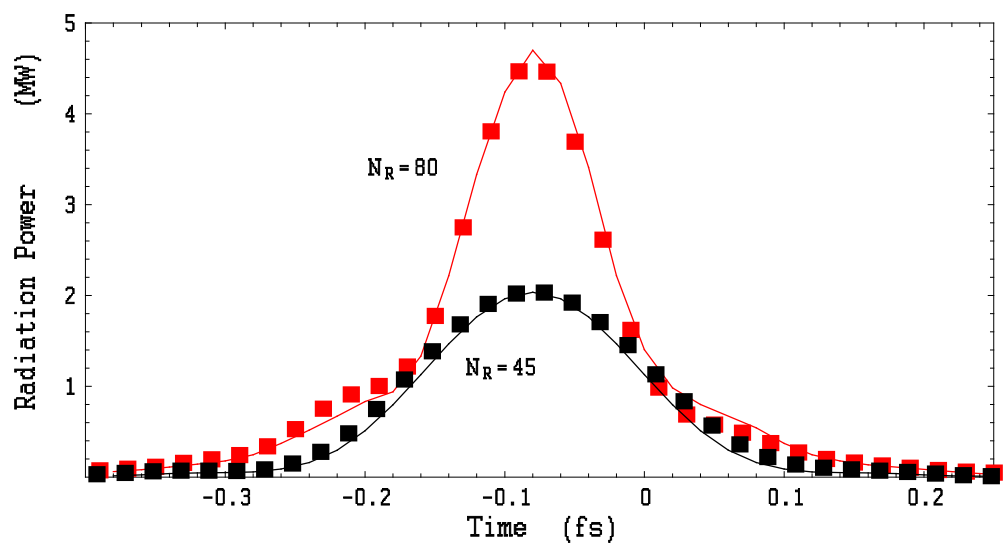

Figure 19. Predicted attosecond pulse power at 1-nm wavelength from a radiator with $N_{R}=80$ (top line) and $N_{R}=45$ (bottom line). The solid line shows analytical prediction and the dots are simulation results from GINGER. Both curves were normalized to the peak intensity of $N_{R}=80$ simulation results.

Those electrons that underwent the 800-nm energy modulation can be strongly bunched at this wavelength via an achromatic bend with a relatively large $R_{56}$ coefficient following the UR (see Fig. 17). Then they will emit a few-cycle, sub-microjoule pulse in a subsequent few-period wiggler magnet shown as $800-\mathrm{nm}$ radiator in Fig. 17. This secondary pulse (which is automatically temporally synchronized with the $\mathrm{x}$-ray attosecond pulse) can then be cross-correlated with the original 800-nm modulating pulse. This measurement can be used for a fine correction of the timing delay between laser pump and x-ray probe pulses that can possibly develop on the electron bunch pass from the 800-nm modulator to the UR.

The contrast of the signal, which is defined as the ratio of energy in the main attosecond peak to the total radiated energy composed from two side peaks (not shown in Fig. 19) plus spontaneous emission background, is of the order of 100. In this estimate it assumed that spontaneous emission background is angular collimated and that a 
double-grating monochromator with a path-length compensation selects an attosecond pulse that is spectrally shifted $\sim 1 \%$ from the central peak of the spontaneous emission.

Another proposal [Sald3] uses a strong, temporally localized energy chirp $d \gamma / d t$ in the center of the sine-like modulation waveform shown in Fig. 16(b). Under normal conditions the energy chirp causes the FEL gain degradation, but it can be prevented by means of the undulator tapering producing $z$ dependence of the undulator parameter $K$. It can be understood by considering that the field experienced by the test electron was emitted by a second electron behind it at a retarded time. It is best when the carrier frequency of this field is in the FEL resonance with the test electron, e.g., when $\gamma^{2}=\lambda_{u} / 2 \lambda_{s} \times\left(1+K^{2} / 2\right)$, where $\lambda_{u}$ is the undulator period and $\lambda_{s}$ is the wavelength of the field. Therefore, the second electron with the energy offset can only emit the field with the right frequency if undulator parameters are different at the retarded time. For large $d \gamma / d t$ this requirement can be formulated with an approximate condition: $d \gamma / c d t \times\left(\bar{\beta}_{z}-1\right) \approx(d \gamma / d K) \times(d K / d z)$, where $\bar{\beta}_{z}$ is the electron longitudinal velocity averaged over the undulator period and normalized on $c$. Equivalently, one can obtain [Sald3, Fawl3]

$$
\frac{d \ln K}{d z}=-\frac{\lambda_{s}}{\lambda_{u}} \frac{1+K^{2} / 2}{K^{2} / 2} \frac{d \ln \gamma}{c d t} .
$$

With the undulator taper matching in Eq. (12), only a short slice of the electron bunch around the zero-crossing of the energy modulation in Fig. 16(b) will produce a powerful FEL pulse. The main unmodulated part of the electron bunch will suffer from the undulator taper and will have much reduced or nonexistent FEL gain. Fig. 20 shows that in fact the calculated output signal is dominated only by one slice of the electron bunch. 
Typical pulse duration of the peak is about 200 attoseconds (FWHM) and typical peak power ranges up to $100 \mathrm{GW}$. The estimated contrast of the collimated attosecond pulse influenced by SASE intensity in two side peaks (barely visible in Fig. 20) and by spontaneous and SASE emission in the first harmonic from the rest of the electrons in the bunch is of the order of one unit [Sald3].

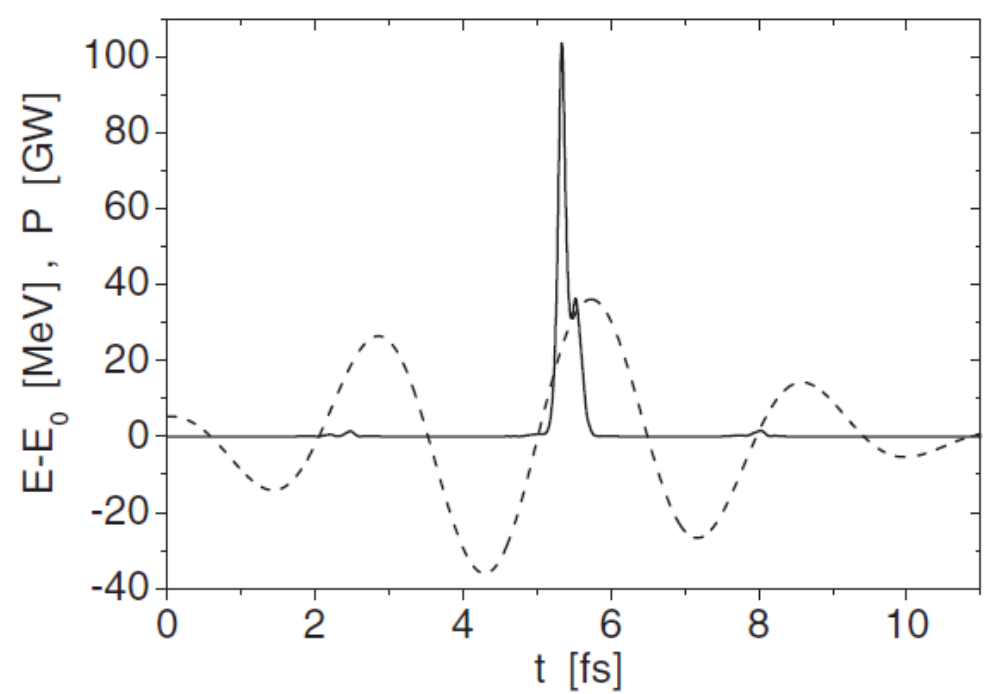

Figure 20. Energy modulation of the electron beam at the exit of the modulator undulator (dotted line) and a profile of the radiation pulse at the exit of the FEL.

The energy chirp within the slice of the electron bunch responsible for the main signal leads to a corresponding frequency chirp in the output signal. Since one can change the magnitude and sign of the energy chirp by manipulating the strength and phase of the few-cycle optical laser pulse (or by combining two or more few-cycle laser pulses similar to [Zhol7, Ding2]), one can vary the strength and sign of the frequency chirp in the x-ray pulse. This opens the possibility to obtain broad-bandwidth pulses with well-characterized frequency chirp that in some cases can probe the attosecond time scale electron dynamics better than ultrashort transform-limited pulses [Yudi]. Studies of a 
“chirp manipulation” in [Fawl3] illustrated on an example of the generation of a 2-fs-long pulse at 8-nm wavelength demonstrate possibilities for large frequency chirp adjustments.

Keeping with the tradition of using a few-cycle CEP-stabilized laser pulse, another technique for generation of attosecond x-ray pulses uses modulation of the electron transverse momentum (angles) instead of energy modulation by employing the laser field with a Hermite-Gaussian TEM $_{10}$ mode [Zhol8]. This laser mode is capable of kicking electrons transversely with an amplitude of $\Delta x_{0}^{\prime} \propto \sqrt{A_{L}} / \gamma^{2}$ when interacting with electrons in the wiggler. Typically, it is not a strong kick. For example, it is weaker by a factor of $\gamma$ than a relative energy change induced by a TEM To $_{0}$ laser mode (see Eq. (2)). However, this kick does not need to be very strong because one can make a small rms angular spread of the electron beam due to a small emittance and large beta function in the wiggler magnet. Thus, it should be possible to induce the angular modulation with an amplitude that exceeds the rms angular spread of electrons by a large factor, as shown in Fig. 21, even with a relatively small kick.

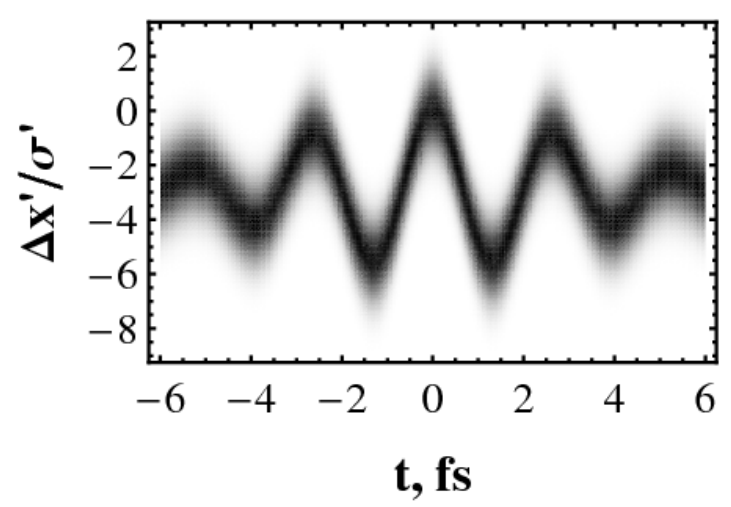

Figure 21. Calculated angular modulation of the $14-\mathrm{GeV}$ electron bunch with the normalized emittance of $10^{-6}$ meters and beta function in the wiggler $\beta_{x 0}=200 \mathrm{~m}$, e.g., $\left.\sigma_{x^{\prime}}=\sqrt{\varepsilon_{x} /\left(\beta_{x 0} \gamma\right.}\right) \approx 4 \times 10^{-7}$, produced via interaction with a 2.5 -mJ, 5-fs, $\mathrm{TEM}_{10}$ mode laser pulse in a wiggler magnet with one period. 
The angular modulation can be used for a generation of attosecond x-ray pulses in the following way. Typically a SASE FEL employs a long undulator where electrons are guided using natural undulator focusing and external focusing. Passing the entire length of the device, they complete one or more betatron oscillations. If the above-discussed angular modulation of the electron beam is applied prior to entering the device, then "modulated" electrons will propagate through it with orbit oscillations relative to the central axis. Then slippage caused by orbit oscillations can influence the FEL gain. For example, because of the slippage, electrons acquire additional phase shift $\Delta \phi \approx k_{x}\left(\Delta x^{\prime}\right)^{2} L_{G} / 2$ with respect to the radiation wave passing one gain length $L_{G}$ in the undulator. Here $k_{x}=2 \pi / \lambda_{x}$, where $\lambda_{x}$ is the x-ray wavelength, and $\left(\Delta x^{\prime}\right)^{2}=\left(\Delta x_{0}^{\prime}\right)^{2} \beta_{x 0} / \bar{\beta}_{x}$ , where $\bar{\beta}_{x}$ is the average beta function in the FEL undulator. The estimate shows that for a large $\Delta x_{0}^{\prime}>\sigma_{x}^{\prime}$, this phase shift can be comparable with the curvature of the radiation wavefront caused by the wave guiding due to the FEL gain. For a quick estimate of the increased gain length one can use the expression

$$
\frac{\Delta L_{G}}{L_{G}} \approx \frac{\Delta \phi / 4}{1-\Delta \phi / 4} .
$$

One can increase the impact of the angular modulation by choosing $\beta_{x 0}>>\bar{\beta}_{x}$. Using $\beta_{x 0}$ $=200 \mathrm{~m}$ and $\bar{\beta}_{x}=20 \mathrm{~m}$, we estimate that orbit oscillations destroy the FEL gain when $\Delta x_{0}^{\prime} \geq 3 \sigma_{x^{\prime}}$. Orbit oscillations also reduce the overlap between the electron distribution and the radiation, and this also affects the FEL gain, but seemingly with a much lesser impact.

Now one can send the electron beam through the wiggler with the angle $-\Delta x_{0}^{\prime}$ and achieve a condition when "unmodulated" electrons propagate the FEL undulator with orbit oscillations and the electrons located at and near the peak of the angular modulation 
without such oscillations. In this situation the entire electron bunch will not lase except for a small part located at and near the peak of the angular modulation. Because these electrons occupy just a fraction of the laser wave cycle, the radiation they produce will be of attosecond duration.

One can also use the same wiggler for energy modulation using a second laser pulse in $\mathrm{TEM}_{00}$ mode to actually combine two modulations at the same time and on the same slice of the electron bunch. The energy modulation can be used to increase the peak current as proposed in [Zhol7, Ding2]. The result, shown in Fig. 22, is a shorter pulse of 115 attosecond (FWHM), higher peak power up to $100 \mathrm{GW}$, and improved contrast of the attosecond pulse that appears to be larger than 100 [Zhol8].

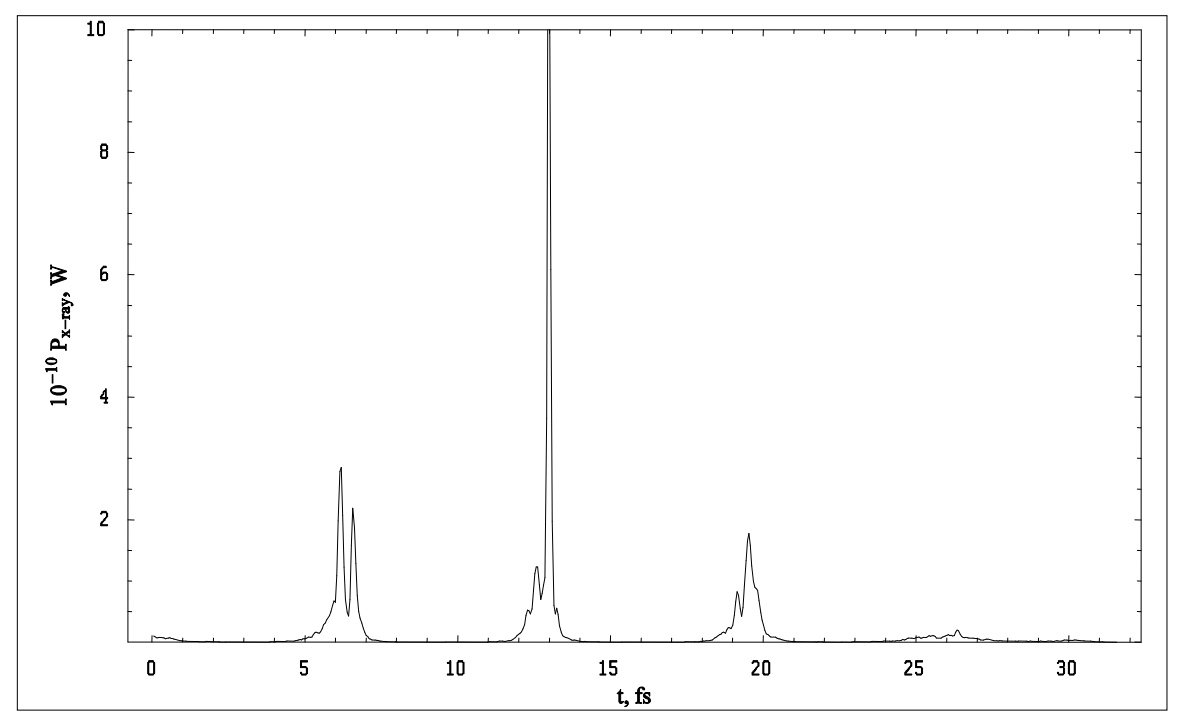

Figure 22. The peak x-ray power calculated for the case of combined angular and energy modulation obtained by simultaneously using a 2- $\mu \mathrm{m}$, CEP-stabilized, 12.5-fs, 0.15 -mJ laser pulse in $\mathrm{TEM}_{00}$ mode and a 2- $\mu \mathrm{m}$, CEP-stabilized, 12.5-fs, 2.5-mJ laser pulse with CEP in $\mathrm{TEM}_{10}$ mode.

The following two methods described here are better suited for a soft x-ray FEL. They both utilize the EEHG seeding technique in order to induce microbunching of electrons at the requisite $\mathrm{x}$-ray wavelength in an ultrashort WS of the electron bunch. 
Then similar to [Zhol6], the electrons in the WS produce coherent radiation in the downstream undulator radiator with attosecond pulse duration that dominates the spontaneous emission of the rest electrons. The scheme depicted in Fig. 23 and proposed in [Xian2] requires a UV seed laser, a few-cycle CEP-stabilized IR laser, together with four undulator sections and two dispersion sections. The wavelength of the UV seed laser is assumed to be $200 \mathrm{~nm}$ and that of the few-cycle IR laser is $800 \mathrm{~nm}$. We further assume that the lasers originate from the same Ti:Sapphire oscillator, which will allow tight synchronization between them at a level of a few hundreds of attoseconds.

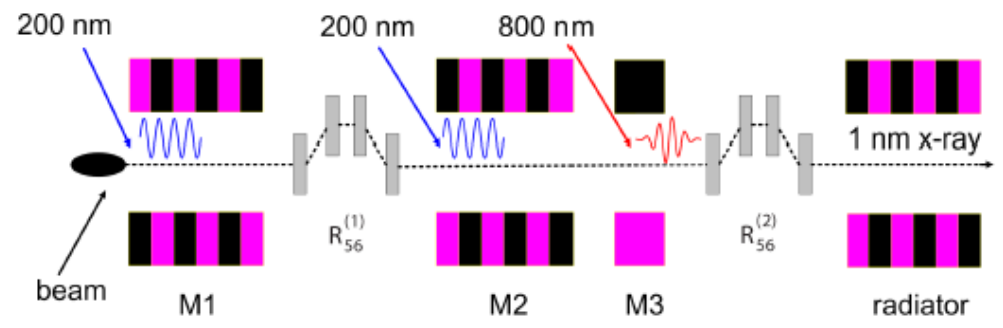

Figure 23. The scheme for generation of an attosecond x-ray pulse proposed in [Xian2].

The beginning of the proposed scheme is the same as in an EEHG FEL [Stup, Xian1]; i.e., the electron bunch is energy modulated in wiggler M1 and then sent through a dispersion section with strong dispersion, after which the modulation obtained in M1 produces separated energy bands in the longitudinal phase space. The second wiggler M2 is used to energy-modulate this bunch, and the second dispersion section is used to convert the separated energy bands into the current modulation, giving rise to electron microbunching at a high harmonic frequency. In this scheme, wiggler M3—containing just two periods—is introduced before the dispersion section. Electrons interact in M3 with a few-cycle laser pulse whose wavelength is chosen to be several times longer than 
that of the laser in M2, so that some of the electrons around the zero crossing of the fewcycle laser get almost linear energy chirp. Then, with this additional energy chirp, the electrons in the WS are longitudinally compressed after passing through the second dispersion section, and the harmonic number is increased by the compression factor. On a large scale, the longitudinal phase space after M3 may look very similar to Fig. 16(b), but it appears with a structure in the phase space that is clearly seen in Fig. 24 on a small scale.

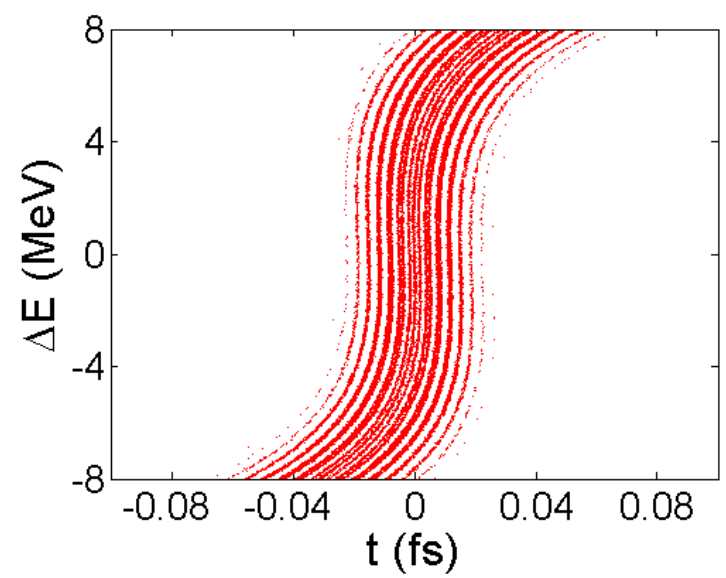

Figure 24. A fragment of the electron bunch longitudinal phase showing microbunching at 1-nm spacing.

Because of the nature of a few-cycle laser pulse and the fine tuning of the dispersion section after M3, this microstructure appears only in one particular area of the WS where the energy chirp is the strongest. This results in a pulse of coherent radiation in the downstream undulator whose FWHM is only 20 attoseconds (see Figure 25), which dominates the spontaneous emission of the rest of the electrons. 


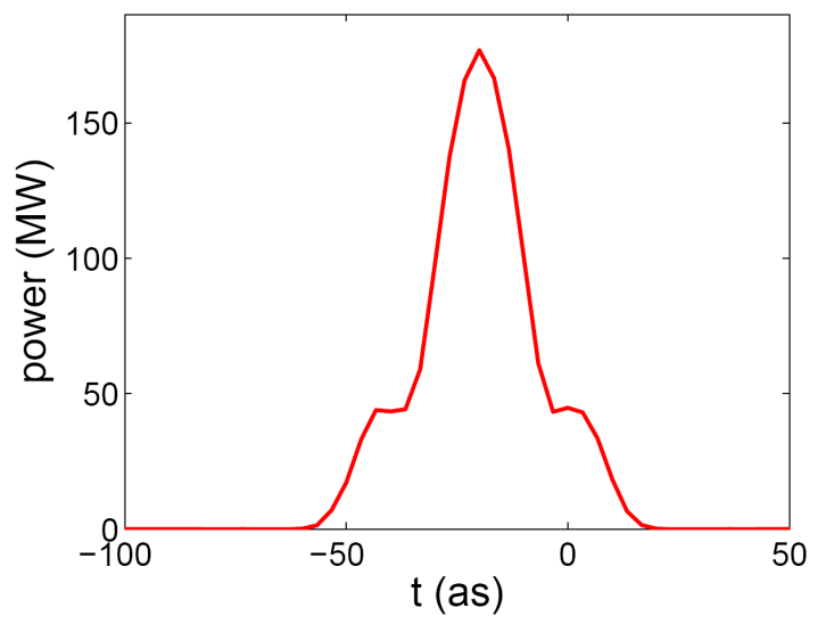

Figure 25. Calculated power of $x$-ray radiation of the electrons in the undulator radiator. The FWHM pulse length is 20 attoseconds.

The next proposal has a slightly different thrust than the previous proposals; namely, it addresses production of a sequence of two attosecond x-ray pulses with two different carrier frequencies and an adjustable time delay between pulses that can be controlled with sub-fs precision [Zhol9]. Figure 26 schematically shows the main idea.

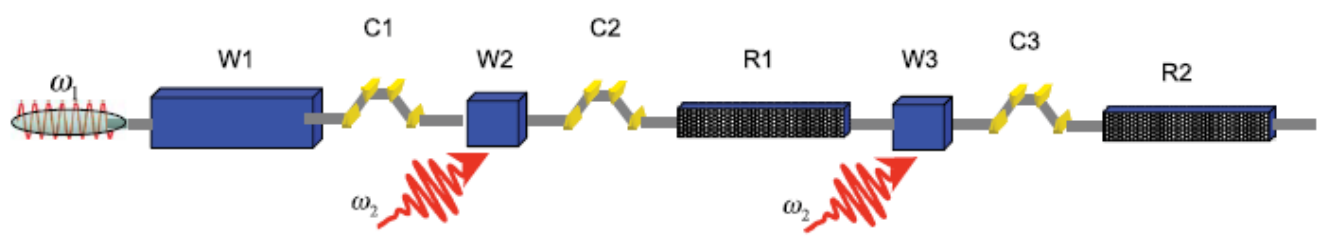

Figure 26. A schematic of the generation of two attosecond x-ray pulses, where W1, W2, and W3 are wiggler magnets; C1, C2, and C3 are dispersion sections; R1 and R2 are $\mathrm{x}$ ray undulator radiators; $\omega_{1}$ is the carrier frequency of the long laser pulse; and $\omega_{2}$ is the carrier frequency of a few-cycle laser pulse that is split into two pulses.

The beginning of the new scheme is the same as the previous scheme, e.g., the electron bunch is energy-modulated in wiggler W1 and then sent through a dispersion section C1, after which the modulation obtained in W1 produces separated energy bands in the longitudinal phase space. In wiggler W2 a few-cycle laser pulse interacts with a 
short WS of the electron bunch and produces a sine-like form of energy modulation with carefully adjusted amplitude. Then, on the basis of this modulation, the following dispersion section C2 enhances the peak current and converts energy bands within a narrow slice of the WS located in the vicinity of a zero-crossing of the energy modulation waveform into the modulation of the peak current and hence produces microbunching. The magnitude of the dispersion in C2 is carefully chosen such that the energy modulation in M2 can be utilized to yield the microbunching at a specific x-ray wavelength $\lambda_{x 1}$. Then electrons bunched at $\lambda_{x 1}$ produce an attosecond pulse of coherent radiation in the downstream undulator R1. The entire process between M2 and R1 is then repeated using a new few-cycle laser pulse and a new short WS of the electron bunch, but this time the amplitude of energy modulation in M3 and the magnitude of the dispersion in C3 are adjusted to yield the microbunching and attosecond pulse in the undulator R2 at a different x-ray wavelength $\lambda_{x 2}$. Since both few-cycle laser pulses can be originated from the same source, the time delay between two laser interactions with the electron bunch in M2 and M3 can be precisely adjusted to yield ultimate control over the time delay between two attosecond x-ray pulses. Moreover, the relative phase of the electric field oscillations in these pulses can also be controlled and well defined. The time delay between two attosecond x-ray pulses can vary from a few fs to as the width of the electron bunch length minus timing jitter in the arrival time of the electron bunch in M2. Figures 27 and 28 show computer simulation results for the above-described scheme wherein the frequency of one attosecond pulses is tuned to the oxygen K-edge and the frequency of the second attosecond pulses is tuned to the nitrogen K-edge. The FWHM length of both pulses is $\sim 200-250$ attoseconds and FWHM bandwidth is $\sim 6-8 \mathrm{eV}$. We 
note that the relative phase of electric field oscillations in both pulses is locked to each other owning to the same original source for a few-cycle laser pulse.

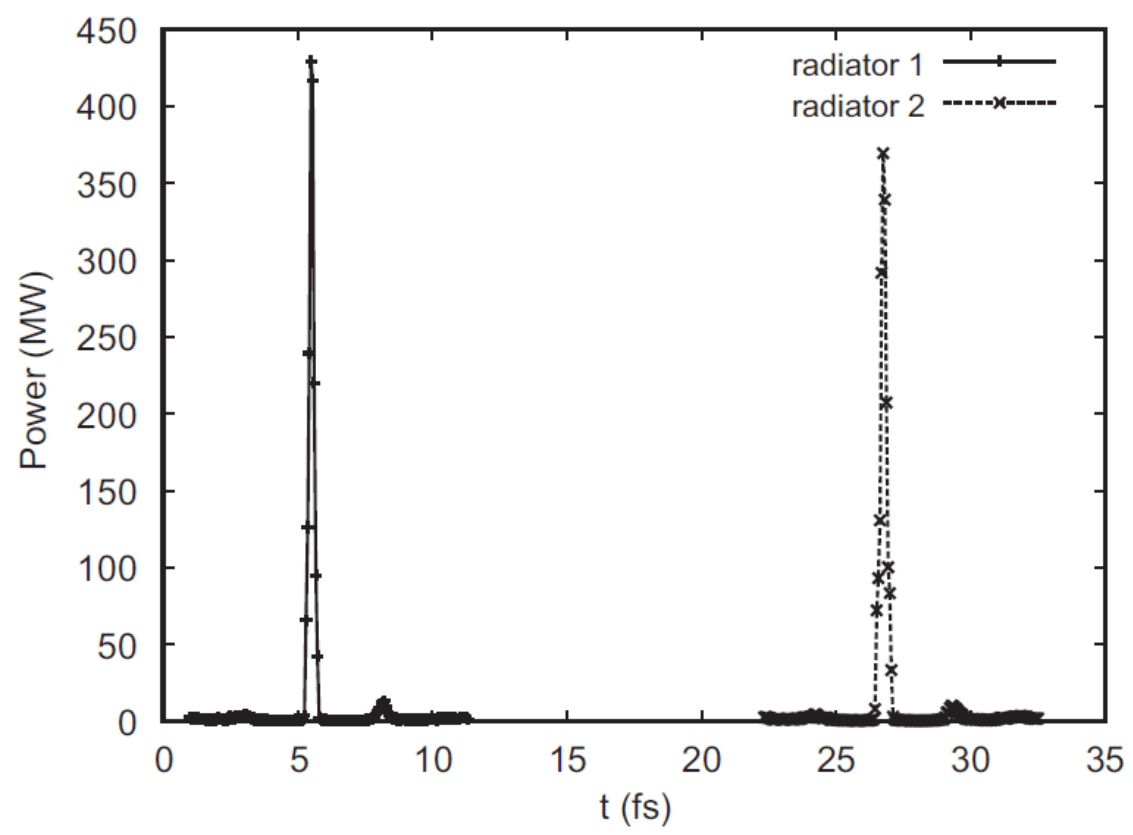

Figure 27. Two x-ray pulses produced in undulators R1 and R2. Coherent radiation of the bunched electrons dominates spontaneous emission.

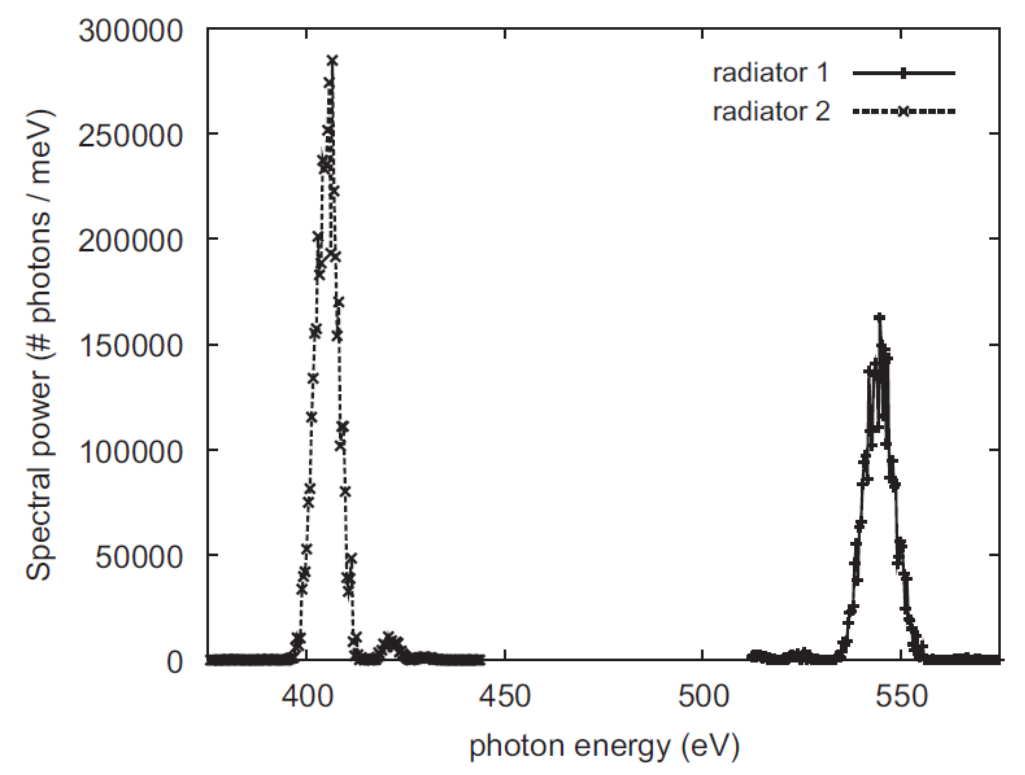

Figure 28. The spectrum of two $\mathrm{x}$-ray pulses produced by the electron bunch radiating in undulators R1 and R2. Pulse intensity is given in units of number of photons per meV bandwidth. 
If an experiment does not need two attosecond x-ray pulses with controlled phases of the electric field oscillations and does not need two different wavelengths, then two xray pulses with the same wavelength can be obtained in a much simpler way. For example, it was proposed in [Emma3] to spoil electron beam emittance everywhere in the electron bunch except a short section. The idea was that this action will impact the FEL gain everywhere in the electron bunch except this section, and, thus, the output signal will consist of only one spike of coherent radiation sitting on the plateau of spontaneous emission coming from the rest of the electrons. This approach can be easily expanded into production of two or more spikes just by leaving two or more unspoiled sections. Preliminary measurements conducted at the LCLS at the time of this writing seem to confirm anticipated results [SLAC]. By varying the locations of the bright spots on the electron bunch, one would then vary the time delay between the x-ray pulses. It is remarkable that with this technique there is no need even to retune the machine switching between operation with and without spoiler since there is no electron beam loss associated with the spoiler.

Another approach advanced in [Rosen] goes even further and suggests taking a short and low-charge electron bunch from the electron gun and compressing it to a sub-fs pulse length by the time the electron bunch reaches the end of the accelerator and enters the FEL. Then, two x-ray pulses, for a pump-probe experiment and adjustable time delay between pulses, can be obtained by first splitting the x-ray pulse produced in the FEL into two parts and then adjusting the path lengths for each part. 
All techniques for generation of ultrashort x-ray pulses discussed so far were focused on obtaining of a solitary pulse (or two pulses when it deemed necessary). However, obtaining a comb-like sequence of ultrashort $\mathrm{x}$-ray pulses is also possible with all the above described techniques involving the laser. Indeed, a long laser pulse will rather naturally assist in creation of one attosecond x-ray pulse at every optical cycle, thus, producing a periodic sequence of $\mathrm{x}$-ray pulses with the total length of the pulse train equal to the length of the laser pulse. Furthermore, a mode-locking technique proposed in [Thom] can be used to reduce the width of each individual pulse in the pulse train down to a few tens of attoseconds.

\section{Outlook}

To the best of our knowledge, generation of sub-ps x-ray pulses by a relativistic electron beam was demonstrated for the first time in 1996 in [Scho4]. This event and subsequent measurement of ultrafast structural dynamics in InSb [Chin] gave birth to the accelerator-based ultrafast x-ray science at the sub-ps time scale. Now, fourteen years later, the state of the art for the generation of ultrashort x-ray pulses using conventional accelerators has moved forward dramatically owing in part to a spectacular development of laser technology, including development of few-cycle laser pulses with a CEP stabilization. Three synchrotron light sources-ALS (http://www.als.lbl.gov), BESSY (http://www.helmholtz-berlin.de), and SLS (http://sls.web.psi.ch)—routinely operate "femtosecond" x-ray beamlines providing users with 100- to 200-fs x-ray pulses of

undulator radiation. The SPPS source of 80-fs $\mathrm{x}$-ray pulses (http://www.slac.stanford.edu/ pkr/SPPS/SPPS.html) lived a short but memorable life 
and then made room for the LCLS FEL. Three other synchrotron light sources SOLEIL (http://www.synchrotron-soleil.fr), Sprin8 (http://www.spring8.or.jp/en) and APS (http://www.aps.anl.gov) — are actively pursuing plans to add ultrafast x-ray science capabilities to their facilities. All three existing x-ray FELs, i.e., FLASH (http://flash.desy.de/), $\quad$ SCSS (http://www.spring8. or.jp), and LCLS (https://slacportal.slac.stanford.edu/sites/lcls_public/), routinely produce $\mathrm{x}$-ray pulses lasting dozens of femtoseconds, or even a few femtoseconds (in the case of LCLS), although to take a full advantage for pump-probe experiments, a high-precision synchronization of the x-ray pulses to external laser sources has yet to be implemented. It is also clear (thanks to a great number of ideas) that it will not take long before attosecond pulses with precise synchronization to the external laser sources and pulse energy at a microjoule level will be routinely produced by FELs. It will also be possible to have groups of two or more attosecond pulses with a controlled time delay between individual pulses and variable wavelengths for individual pulses. These new emerging tools will assist studies of matter that then will be carried out with an unprecedented time and spatial resolution.

I gratefully acknowledge that during the many years I have been interested in the problem of generation of ultrashort $\mathrm{x}$-ray pulses I have had the pleasure to discuss it with many people including M. Borland, J. Byrd, S. Chattopadhyay, M. Cornacchia, P. Emma, P. Heimann, S. Khan, K.-J. Kim, K. Holldack, Z. Huang, C. Pellegrini, G. Penn, R. Schoenlein, A. Sessler, V. Sajaev, G. Stupakov, D. Xiang, M. Zolotorev. This work was supported by the U.S. Department of Energy, Office of Science, Office of Basic Energy Sciences, under Contract No. DE-AC02-06CH11357. 


\section{References:}

[Acke] W. Ackermann et al., "Operation of a free-electron laser from the extreme ultraviolet to the water window”, Nature Photonics 1, 336, 2007.

[Alfe] D.E. Alferov, Y.A. Bushmakov, K.A. Belovintsev, E.G. Bessonov, and P.A. Cherenkov, Particle Accel. 9, 223, 1979.

[Alte] D. Altenbernd, U. Teubner, P. Gibbon, E. Förster, P. Audebert, J.-P. Geindre, J.C. Gauthier, G. Grillon, and A. Antonetti, "Soft x-ray brilliance of femtosecond and picosecond laser-plasmas.,” J. Phys. B 30, 3969, 1997.

[Amir] A. Amir and Y. Greenzweig, "Three-dimensional free electron laser gain and evolution of optical modes,” Nucl. Instrum. Methods in Phys. Res. A 250, 404, 1986.

[Ande] T. Anderson, I.V. Tomov, and P.M. Rentzepis, "Laser-driven metal photocathodes for picosecond electron and x-ray pulse generation,” J. Appl. Phys. 71, 516, 1992.

[Arpi] P. Arpin, T. Popmintchev, N.L. Wagner, A.L. Lytle, O. Cohen, H.C. Kapteyn, M.M. Murnane, "Enhanced high harmonic generation from multiply-ionized argon above $500 \mathrm{eV}$ through laser pulse self compression”, Phys. Rev. Lett. 103, 143901, 2009.

[Arut] F.R. Arutyunian and V.A. Tumanian, "The Compton effect on relativistic electrons and the possibility of obtaining high energy beams," Phys. Lett. 4, 176, 1963.

[Balt] A. Baltuška, Th. Udem, M. Uiberacker, M. Hentschel, E. Goulielmakis, Ch. Gohle, R. Holzwarth, V. S. Yakovlev, A. Scrinzi, T. W. Hänsch and F. Krausz, "Attosecond control of electronicprocesses by intense light fields”, Nature 421, 611, 2003.

[Bane] K.L.F. Bane and P. B. Wilson, "Bunch lengthening due to potential well distortion from cylindrical cavities with beam ports”, IEEE Trans. Nucl. Sci. NS24(3), 1485, (1977).

[BES1] U.S. Department of Energy, Basic Energy Sciences Advisory Committee, "Directing Matter and Energy: Five Challenges for Science and the Imagination," http://www.er.doe.gov/bes/reports/list.html, 2007.

[BES2] U.S. Department of Energy, Basic Energy Sciences Advisory Committee, "NextGeneration Photon Sources for Grand Challenges in Science and Energy,” http://www.er.doe.gov/bes/reports/list.html, 2009.

[BES3] U.S. Department of Energy, Basic Energy Sciences Advisory Committee, "New Science for a Secure and Sustainable Energy Future,” http://www.er.doe.gov/bes/reports/list.html, 2009.

[Brab] T. Brabec and F. Krausz, "Intense few-cycle laser fields: Frontiers of nonlinear optics”, Rev. Mod. Phys. 72, 545, 2000.

[Borl1] M. Borland provided the data for plots in Figure 11 and also made Figure 12. As a specific example, the design study for an upgrade of the APS was used.

[Borl2] M. Borland, "Simulation and analysis of using deflecting cavities to produce short x-ray pulses with the Advanced Photon Source”, Phys. Rev. ST Accel. Beams, 8, 074001, 2005. 
[Borl3] M. Borland and V. Sajaev, "Simulations of x-ray slicing and compression using crab cavities in the advanced photon source”, Proc. of the 2005 Particle Accelerator Conference, Knoxville, 3888, 2005; http://www.JACoW.org.

[Byrd1] J. Byrd, Z. Hao, M. Martin, D. Robin, F. Sannibale, R. Schoenlein, A. Zholents, and M. Zolotorev, "Tailored Terahertz Pulses from a Laser-Modulated Electron Beam”, Phys. Rev. Lett. 96, 164801, 2006.

[Byrd2] J.M. Byrd, L.R. Doolittle, G. Huang, J.W. Staples, R.B. Wilcox, J. Arthur, J.C. Frisch, W.E. White, "Femtosecond synchronization of laser systems for the LCLS”, Proc. of the 2010 International Particle Accelerator Conference, Kyoto, Japan, 58, 2010; http://www.JACoW.org.

[Cava] A.L. Cavalieri et al., “Clocking Femtosecond X Rays”, PRL 94, 114801, 2005.

[Chao] A.W. Chao, in Physics of Collective Beam Instabilities in High Energy Accelerators, (Wiley, New York: 1993).

[Chin] A. H. Chin, R. W. Schoenlein, T. E. Glover, P. Balling, W. P. Leemans, and C. V. Shank, "Ultrafast structural dynamics in InSb probed by time-resolved X-ray diffraction”, Phys. Rev. Lett., 83, 336(1999).

[Chur] For grazing-incidence optics, in-plane scattering dominates over out-of-plane scattering due to the length scales of roughness sampled, see: E. L. Church and P. Z. Takacs, in Handbook of Optics, Vol. 1, pp. 7.1-7.14 (McGraw-Hill, Inc.: 1975).

[Corn] M. Cornacchia et al., “A Subpicosecond Photon Pulse Facility for SLAC”, SLACPUB-8950, LCLS-TN-01-7, 2001.

[Cson] P. Csonka, Part. Accel. 11, 45, 1980.

[Ding1] Y. Ding et al. , "Measurements and Simulations of Ultralow Emittance and Ultrashort Electron Beams in the Linac Coherent Light Source", Phys Rev Lett. 102, 254801, 2009.

[Ding2] Y. Ding, Z. Huang, D. Ratner, P. Bucksbaum and H. Merdji, "Generation of attosecond X-ray pulses with a multicycle two-color enhanced self-amplified spontaneous emission scheme”, Phys. Rev. ST Accel. Beams 12, 060703, 2009.

[Elia] L.R. Elias, W.M. Fairbank, J.M.J. Madey, H.A. Schwettman, and T.I. Smith, "Observation of Stimulated Emission of Radiation by Relativistic Electrons in a Spatially Periodic Transverse Magnetic Field”, Phys. Rev. Lett., 36, 717, 1976.

[Emma] P. Emma, "Issues and challenges for short pulse radiation production", Proc. of the 2004 European Particle Accelerator Conference, Lucerne, Switzerland, 225, 2004; http://www.JACoW.org.

[Emma2] P. Emma et al., "First lasing and operation of an angstrom-wavelength freeelectron laser”, Nature Photonics, 4, 641, 2010.

[Emma3] P. Emma, K. Bane, M. Cornacchia, Z. Huang, H. Schlarb, G. Stupakov, D. Walz, "Femtosecond and subfemtosecond x-ray pulses from a self-amplified spontaneous-emission-based free-electron Laser," Phys. Rev. Lett. 92, 74801, 2004.

[Fawl1] W. Fawley et al., "X-ray laser seeding for short pulses and narrow bandwidth", Proc. of the 2003 Particle Accelerator Conference, Portland, Oregon, 923, 2003 ; http://www.JACoW.org.

[Fawl2] W.M. Fawley, LBNL Technical Report 49625, 2001.

[Fawl3] W.M. Fawley, "Production of ultrashort FEL XUV pulses via a reverse undulator taper,” Nucl. Instrum. Methods A 593, 111, 2008. 
[Feik1] J. Feikes, K. Holldack, P. Kuske, G. Wüstefeld, "Sub-picosecond electron bunches in the BESSY storage ring”, Proc. of the 2004 European Particle Accelerator Conference, Lucerne, Switzerland, 2290, 2004; http://www.JACoW.org.

[Feik2] J. Feikes, M. von Hartrott, G. Wüstefeld, A. Hoehl, R. Klein, R. M“uller, G. Ulm, "Low alpha operation of the MLS electron storage ring”, Proc. of the 2009 Particle Accelerator Conference, Vancouver, BC, Canada, (2009); to be published.

[Fuji] T. Fujita et al., "Overview of short-pulse x-ray generation using crab cavities at Spring-8”, Proc. of the 2010 International Particle Accelerator Conference, Kyoto, Japan, MOOCMH02, 39, 2010; http://www.JACoW.org.

[Gao] J. Gao, "Bunch lengthening and energy spread increasing in electron storage rings", Nucl. Instrum. Methods in Phys. Res. A 418, 332, 1998.

[Ghas] H. Ghasem, G. H. Luo, A. Mohammadzadeh, Proc. of the 2010 International Particle Accelerator Conference, Kyoto, Japan, WEPEA025, 2538, (2010); http://www.JACoW.org.

[Holl] K. Holldack, S. Khan, R. Mitzner, T. Quas, "Femtosecond Terahertz Radiation from Femtoslicing at BESSY”, Phys. Rev. Lett. 96, 054801, 2006.

[IEEE] IEEE Journal of Selected Topics in Quantum Electronics 9, issue 4, 2003.

[Itat] J. Itatani, F. Quéré, G. Yudin, M. Ivanov, F. Krausz, P. Corkum, “Attosecond Streak Camera” Phys. Rev. Lett. 88, 173903, 2002.

[Jone] D. Jones, S. Diddams, J. Ranka, A. Stenz, R. Windeler, J. Hall, S. Cundiff, "Carrier-envelope phase control of femtosecond mode-locked lasers and direct optical frequency synthesis”, Science 288, 635, 2000.

[Kato] M. Katoh, "Ultra-short pulses of synchrotron radiation on storage rings”, Jpn. J. Appl. Phys. 38, 547, 1999.

[Kim1] K.-J. Kim, S. Chattopadhyay, and C. V. Shank, "Generation of femtosecond xray pulses by 90 degree Thomson scattering," Nucl. Instrum. Method in Phys. Res. A 341, 351, 1994.

[Kim2] K.-J. Kim, “Physics of Particle Accelerators”, AIP Conf. Proc. 184, 565, 1989.

[KimJ] J. Kim, J. Cox, J. Che, F. Kärtner, "Drift-free femtosecond timing synchronization of remote optical and microwave sources”, Nature Photonics, 2, 733, 2008.

[Khan] S. Khan, K. Holldack, T. Kachel, R. Mitzner, T. Quast, "Femtosecond Undulator Radiation from Sliced Electron Bunches”, Phys. Rev. Lett. 97, 074801, 2006.

[Klai] M. Klaiber, K.Z. Hatsagortsyan, C. Müller and C.H. Keitel, Coherent hard x rays from attosecond pulse train-assisted harmonic generation, Optics Lett. 33, 411-413 (2008).

[Krau] F. Krausz and M. Ivanov, Rev. Mod. Phys. 81, 163, 2009.

[Krej] P. Krejcik, F.-J. Decker, P. Emma, K. Hacker, L. Hendrickson, C.L. O’Connell, H. Schlarb, H. Smith, M. Stanek, "Commissioning of the SPPS linac bunch compressor," Proc. of the 2003 Particle Accelerator Conference, Portland, Oregon, 423, 2003; http://www.JACoW.org.

[Lawr] Lawrence Berkeley National Laboratory, “Toward Control of Matter: Energy Science Needs for a New Class of X-Ray Light Sources,” 2008; https://hpcrd.lbl.gov/sxls/home.html. 
[Lamb] G. Lambert et al., "Injection of harmonics generated in gas in a free-electron laser providing intense and coherent extreme-ultraviolet light”, Nature Physics 4, 296, 2008.

[Limb] C. Limborg, "Ultimate brilliance of storage ring based synchrotron facilities of the 3-rd generation”, PhD Thesis, University Joseph Fourier, Grenoble, France, 1996; "Present time structure properties of storage ring based x-ray sources," presented at Time Structure of X-ray Sources and its Applications, San Diego, CA, SPIE, 1998.

[Lind1]A.M. Lindenberg, I. Kang, S.L. Johnson, T. Missalla, P.A. Heimann, Z. Chang, J. Larsson, P.H. Bucksbaum, H.C. Kapteyn, H.A. Padmore, R.W. Lee, J.S. Wark, and R.W. Falcone, "Time-resolved x-ray diffraction from coherent phonons during laserinduced phase transition,”, Phys. Rev. Lett. 84, 111, 2000.

[Lind2] A.M. Lindenberg, et al., "Atomic-Scale Visualization of Inertial Dynamics", Science 308, 392 (2005).

[Leem] W.P. Leemans, R.W. Schoenlein, P. Volfbeyn, A.H. Chin, T.E. Glover, P. Balling, M. Zolotorev, K.-J. Kim, S. Chattopadhyay, and C. V. Shank, "X-ray based time resolved electron beam characterization via $90^{\circ}$ Thomson scattering," Phys. Rev. Lett. 77, 4182, 1996.

[Löhl] F. Löhl et al., "Electron Bunch Timing with Femtosecond Precision in a Superconducting Free-Electron Laser”, Phys. Rev. Lett., 104, 144801, 2010.

[Made1] J.M.J. Madey, "Stimulated emission of bremsstrahlung in a periodic magnetic field”, J. Appl. Phys. 42, 1906, 1971.

[Made2] J.M.J. Madey, Nuovo Cimento 50B, 64, 1979.

[Milb] R.H. Milburn, "Electron scattering by an intense polarized photon field," Phys. Rev. Lett. 10, 75, 1963.

[Morp] J.B. Morphy, C. Pellegrini, in Laser Handbook, Vol. 6, p. 9, (Elsevier Science;1990).

[Murn] M.M. Murnane, H.C. Kapteyn, M.D. Rosen, R.W. Falcone, "Ultrafast pulses from laser-produced plasmas", Science 251, 531, 1991.

[Nadj] A. Nadji et al., "Femtosecond electron beam slicing project at SOLEIL", Proc. of the 2004 European Particle Accelerator Conference, Lucerne, Switzerland, 2332, 2004; http://www.JACoW.org.

[Pell] C. Pellegrini and A.M. Sessler, Nuovo Cimento 3, 116, 1971.

[Penn1] G. Penn, M. Reinsch, J. Wurtelet, J.N. Corlett, W.M. Fawley, A. Zholents, W. Wan, "Harmonic cascade FEL designs for LUX", Proc. of the 2004 European Particle Accelerator Conference, Lucerne, Switzerland, 488, 2004; http://www.JACoW.org.

[Penn2] G. Penn, "Staged Energy Cascade for the LUX FEL”, LBNL-44973, 2004.

[Risc] C. Rischel, A. Rousse, I. Uschmann, P.-A. Albouy, J.-P. Geindre, P. Audebert, J.C. Gauthier, E. Forster, J.-L. Martin, and A. Antonetti, "Femtosecond time-resolved X-ray diffraction from laser-heated organic films,” Nature 390, 490, 1997.

[Rose] C. Rose-Petruck, R. Jimenez, T. Guo, A. Cavalleri, C. Siders, F. Raksi, J.A. Squier, B.C. Walker, K.R. Wilson, and C.P.J. Barty, "Picosecond-milliangstrom lattice dynamics measured by ultrafast x-ray diffraction,” Nature 398, 310, 1999.

[Rosen] J.B. Rosenzweig et al., "Generation of Ultra-Short, High Brightness Electron Beams for Single Spike SASE FEL Operation” Nucl. Instrum. Methods A 593, 39 (2008). 
[Rund] A. Rundquist, C.G. Durfee, Z. Chang, C. Herne, S. Backus, M.M. Murnane, and H.C. Kapteyn, "Phase-matched generation of coherent soft X-rays," Science 280, 1412, 1998.

[Saja] V. Sajaev and A.A. Zholents, LBNL, unpublished information, 2007.

[Sald1] E.L.Saldin, E.A.Schneidmiller, and M.V.Yurkov, “Terawatt-scale sub-10-fs laser technology -key to generation of GW-level attosecond pulses in X-ray free electron laser”, Opt. Comm. 237, 153, 2004.

[Sald2] E.L.Saldin, E.A.Schneidmiller, and M.V.Yurkov, "A new technique to generate 100 GW-level attosecond X-ray pulses from the X-ray SASE FELs”, Opt. Comm. 239, 161, 2004.

[Sald3] E.L. Saldin, E.A. Schneidmiller, and M.V. Yurkov, "Self-amplified spontaneous emission FEL with energy-chirped electron beam and its application for generation of attosecond x-ray pulses”, Phys. Rev. ST Accel. Beams 9, 050702, 2006.

[Sart] S. Sartania, Z. Cheng, M. Lenzner, G. Tempea, Ch. Spielmann, F. Krausz, and K. Ferencz, "Generation of 0.1-TW 5-fs optical pulses at a 1-kHz repetition rate", Opt. Lett. 22, 1562, 1997.

[Schl] V. Schlott, D. Abramsohn, G. Ingold, P. Lerch, P. Beaud, “THz diagnostic for the femtosecond bunch slicing project at the swiss light source", Proc. of the 2004 European Particle Accelerator Conference, Edinburg, Scotland, TUPCH094, 1229, 2006; http://www.JACoW.org.

[Schn] M. Schnurer, C. Spielmann, P. Wobrauschek, C. Streli, N. H. Burnett, C. Kan, K. Ferencz, R. Koppitsch, Z. Cheng, T. Brabec, and F. Krausz, "Coherent 0.5-keV XRay Emission from Helium Driven by a Sub-10-fs Laser”, Phys. Rev. Lett. 80, 3236, 1998.

[Scho1] R.W. Schoenlein, S. Chattopadhyay, H.H. W. Chong, T.E. Glover, P.A. Heimann, C.V. Shank, A. Zholents, and M. Zolotorev, "Generation of x-ray pulses via laser-electron beam interaction,” Appl. Phys. B 71, 1, 2000.

[Scho2] R. Schoenlein, H. Chong, T. E. Glover, P. Heimann, A. Zholents, and M. Zolotorev, Proc. of the 1999 Particle Accelerator Conference, "Low signal FEL gain: Measurement, simulation and analysis," Particle Accelerator Conference, New York, NY, 2498, 1999; http://www.JACoW.org.

[Scho3] R.W. Schoenlein, S. Chattopadhyay, H.H.W. Chong, T.E. Glover, P.A. Heimann, C.V. Shank, A. Zholents, and M. Zolotorev, "Generation of x-ray pulses via laserelectron beam interaction,” Science 287, 2237, 2000.

[Scho4] R.W. Schoenlein, W.P. Leemans, A.H. Chin, P. Volfbeyn, T.E. Glover, P. Balling, M. Zolotorev, K.-J. Kim, S. Chattopadhyay, and C.V. Shank, "Femtosecond $\mathrm{X}$-ray pulses at 0.4 angstroms generated by $90^{\circ}$ Thomson scattering: A tool for probing the structural dynamics of materials,” Science 274, 236, 1996.

[Schr] S. Schreiber, B. Faatz, J. Feldhaus, K. Honkavaara, R. Treusch, “FEL user facility Flash”, Proc. of the 2010 International Particle Accelerator Conference, Kyoto, Japan, 2149, 2010; http://www.JACoW.org.

[Sing] K. P. Singh, F. He, P. Ranitovic, W. Cao, S. De, D. Ray, S. Chen, U. Thumm, A. Becker, M. M. Murnane, H. C. Kapteyn, I.V. Litvinyuk, and C. L. Cocke, Control of Electron Localization in Deuterium Molecular Ions using an Attosecond Pulse Train and a Many-Cycle Infrared Pulse, PRL 104, 023001 (2010).

[SLAC] SLAC Today, July 27, 2010; http://today.slac.stanford.edu/a/2010/07-27.htm 
[Stup] G. Stupakov, "Using the Beam-Echo Effect for Generation of ShortWavelength Radiation”, Phys. Rev. Lett. 102, 074801, 2009.

[Stei] C. Steier, D. Robin, F. Sannibale, R. Schoenlein, W. Wan, W. Wittmer, A. Zholents, "The new undulator based fs-slicing beamline at the ALS", Proc. of the 2005 Particle Accelerator Conference, Knoxville, Tennessee, RPAE082, 4096, 2005; http://www.JACoW.org.

[Thom] N.R. Thompson, B.W.J. McNeil, "Mode Locking in a Free-Electron Laser Amplifier”, Phys. Rev. Lett. 100, 203901, 2008.

[Uesa] M. Uesaka, H. Kotaki, K. Nakajima, H. Harano, K. Kinoshita, T. Watanabe!, T. Ueda, K. Yoshii, M. Kando, H. Dewa, S. Kondo, F. Sakai, "Generation and application of femtosecond X-ray pulse”, Nucl. Instrum. Methods in Phys. Res. A 455, 90, 2000.

[Wang] Y. Wang, E. Granados, F. Pedaci, D. Alessi, B. Luther, M. Berrill, and J.J. Rocca, "Phase coherent table top soft $\mathrm{x}$-ray lasers at $18.9 \mathrm{~nm}$ and $13.9 \mathrm{~nm}$ by saturated amplification of high harmonic pulses in Nickel-like ions," Nature Photonics 2, 94, 2008.

[Wilc] R. Wilcox, J. M. Byrd, L. Doolittle, G. Huang, and J. W. Staples, "Stable transmission of radio frequency signals on fiber links using interferometric delay sensing”, Opt. Lett. 34, 3050, 2009.

[Whit] White paper, "Scientific needs for future x-ray sources", Publication ANL-08/39; BNL-81895; LBNL-1090E; SLAC-R-97, 2008; http://www.als.lbl.gov/als/publications/genpubs.html.

[Wulf] M. Wulff, F. Schotte, G.Naylor, D. Bourgeois, K. Moffat, and G. Mourou, "Timeresolved structures of macromolecules at the ESRF: Single pulse Laue diffraction, stroboscopic data collection and femtosecond flash photolysis," Nucl. Instrum. Methods in Phys. Res. A 398, 69, 1997.

[Wüst] G. Wüstefeld, "Short bunches in electron storage rings and coherent synchrotron radiation”, Proc. of the 2008 European Particle Accelerator Conference, Genoa, Italy, 26, 2008; http://www.JACoW.org.

[Xian1] D. Xiang and G. Stupakov, "Echo-enabled harmonic generation free-electron laser”, Phys. Rev. ST Accel. Beams 12, 030702, 2009.

[Xian2] D. Xiang, Z. Huang and G. Stupakov, "Generation of intense arrosecond x-ray pulses using ultraviolet laser induced microbunching in electron beam”, Phys. Rev. ST Accel. Beams 12, 060701, 2009.

[Yang] J. Yang., M. Yorozu, F. Sakai, T. Yanagida, Y. Okada, T. Nakajyo, S. Ito, K. Takasago, A. Endo, "Spatial profile measurement of femtosecond laser - Compton Xrays", Proc. of the 2002 European Particle Accelerator Conference, Paris, France, 784, 2002; http://www.JACoW.org.

[Yu] L.H. Yu, "Generation of intence UV radiation by subharmonically seeded singlepass free-electron lasaers", Phys. Rev. A44, 5178, 1991.

[Yudi] G.L. Yudin, A.D. Bandrauk, P.B. Corkum, "Chirped Attosecond Photoelectron Spectroscopy”, Phys. Rev, Lett. 96, 063002, 2006.

[Zamp] F. Zamponi, Z. Ansari, C. V. Korff, Schmising, P. Rothhardt, N. Zhavoronkov, M. Woerner, T. Elsaesser, M. Bargheer, T. Trobitzsch-Ryll, M. Haschke, "Femtosecond hard X-ray plasma sources with a kilohertz repetition rate", Appl Phys A 96, 51, 2009. 
[Zewa] Ahmed Zewail received the 1999 Nobel Prize in Chemistry for his studies of the transition reactions using femtosecond spectroscopy. See also "Femtochemistry: Atomic-Scale Dynamics of the Chemical Bond", J. Phys. Chem. A 24, 104, 2000.

[Zhol1] A. A. Zholents and M. S. Zolotorev, "Femtosecond x-ray pulses of synchrotron radiation,” Phys. Rev. Lett. 76, 912, 1996.

[Zhol2] A.A. Zholents, K. Holldack, "Energy modulation of the electrons by the laser field in the wiggler magnet: analysis and experiment", Proc. of the 2006 Free Electron Laser Conference, Berlin, Germany, 725, 2006; http://www.JACoW.org.

[Zhol3] A. Zholents, P. Heimann, M. Zolotorev, J. Byrd, "Generation of subpicosecond X-ray pulse using RF orbit deflection”, Nucl. Instrum. Methods in Phys. Res. A 425, 385, 1999.

[Zhol4] A. Zholents, "Method of an Enhanced Self-Amplified Spontaneous Emission for X-ray Free Electron Lasers”, Phys. Rev. ST Accel. Beams 8, 040701, 2005.

[Zhol5] A. Zholents, W. Fawley, P. Emma, Z. Huang, G. Stupakov, S. Reiche, "CurrentEnhanced SASE Using an Optical Laser and its Application to the LCLC", Proc. of the 2004 Free Electron Laser Conference, Trieste, Italy, 582, 2004; http://www.JACoW.org.

[Zhol6] A. Zholents, W. Fawley, "Proposal for Intense Attosecond Radiation from an XRay Free-Electron Laser”, Phys. Rev. Lett. 92, 224801, 2004.

[Zhol7] A. Zholents and G. Penn, "Obtaining Attosecond X-ray Pulses Using a SelfAmplified Spontaneous Emission Free Electron Laser”, Phys. Rev. ST Accel. Beams 8, 050704, 2005.

[Zhol8] A. Zholents and M. Zolotorev, "Attosecond x-ray pulses produced by ultra short transverse slicing via laser electron beam interaction”, New J. Phys, 10, 025005, 2008.

[Zhol9] A. Zholents, G. Penn, "Obtaining two attosecond pulses for x-ray stimulated Raman spectroscopy”, Nucl. Instrum. Methods in Phys. Res. A 612, 254, 2010. 